\title{
Resonant modulation of the flow in a tidal channel
}

\author{
Julia C. Mullarney, ${ }^{1}$ Alex E. Hay, ${ }^{1}$ and Anthony J. Bowen ${ }^{1}$ \\ Received 24 August 2007; revised 26 June 2008; accepted 17 July 2008; published 11 October 2008.
}

[1] The coupling between a quarter-wave resonance in a coastal bay and a Helmholtz mode in an adjacent cove (connected to the bay through a narrow channel) is investigated by comparing field measurements to analytical and numerical model predictions. Pressure and velocity spectra from locations throughout the bay reveal an oscillation with a period of approximately 1 hour, consistent with a quarter-wave seiche mode. The associated sea surface displacements throughout the bay are small (less than $5 \mathrm{~cm}$ RMS, i.e., only $10 \%$ of the tidal elevation). Velocities within the channel are significantly modulated in the 1-hour band, with amplitudes up to $40 \%$ of the peak tidal current. The analytical model shows that the modulation of the channel flow results from the interaction between the quarter-wave mode in the main basin and a Helmholtz resonance in the cove, also with a period near 1 hour. The amplitude and phase of the 1-hour oscillation varies through the tidal cycle because of the change in Helmholtz frequency with tidal elevation. Good quantitative agreement between the data and the model predictions is obtained if a drag coefficient approximately 3-4 times larger than the classical value of $3 \times 10^{-3}$ is used in the channel and cove.

Citation: Mullarney, J. C., A. E. Hay, and A. J. Bowen (2008), Resonant modulation of the flow in a tidal channel, J. Geophys. Res., 113, C10007, doi:10.1029/2007JC004522.

\section{Introduction}

[2] Oscillations or seiche motions are ubiquitous features in coastal bays and harbors [Miles, 1974]. Often, they are excited by atmospheric disturbances [e.g., Munk et al., 1956; Tintoré et al., 1988; Gomis et al., 1993; de Jong et al., 2003; de Jong and Battjes, 2004]. This paper explores the effects of such an oscillation, which also forces a Helmholtz resonance in an adjacent cove, on the dynamics of the flow within a tidal channel in Lunenburg Bay, Nova Scotia. The primary oscillation, which has approximately 1-hour period, appears to be an amplification of broadbanded atmospheric forcing at the quarter-wave frequency of the bay. This broadbanded forcing exists throughout the year, is strongest in winter and appears to drive amplified sea level variances (on timescales of hours) in all harbors with permanent tide gauges along the Atlantic coast of Canada.

[3] Lunenburg Bay, located on the south eastern coast of Nova Scotia (Figure 1), is the site of an interdisciplinary coastal observatory with permanent moorings recording hydrodynamic, atmospheric and biological data. The bay is shallow and is linked to two adjacent coves through a relatively narrow channel (Corkum's Channel (Figure 2)). During the ebb phase of the tide, water flowing from the coves through the channel generates a strong jet in the main bay with maximum velocities reaching $1 \mathrm{~m} \mathrm{~s}^{-1}$. This jet dominates flows within the bay interior and is thought to serve as an important mechanism for mixing. Observations

\footnotetext{
${ }^{1}$ Department of Oceanography, Dalhousie University, Halifax, Nova Scotia, Canada.

Copyright 2008 by the American Geophysical Union. 0148-0227/08/2007JC004522\$09.00
}

indicate a significant modulation of the flow in the channel, with approximately 1-hour period and amplitude approximately $40 \%$ of the peak tidal current. It is shown that the quarter-wave period of the main bay is close to the period of the Helmholtz resonance of one of the coves and it is the interaction between the two resonant modes, which causes this significant modulation of the tidal current in the channel.

[4] A linear analytical model reproduces the primary features of the modulation (i.e., its amplitude and phase along the channel), thus identifying the coupling between the quarter-wave mode in the bay and the Helmholtz resonance in the cove as the principal physical process. The model includes tidal elevation, which is important because it significantly affects the Helmholtz period, but uses linearized bottom friction, uniform channel geometry, and does not include nonlinear inertial terms. We implement a fully nonlinear numerical model with realistic bathymetry to investigate the importance of these effects. An earlier field experiment made direct measurements of the Reynolds stress in the channel and it was found that the coefficient of friction in the channel was 3 to 4 times larger than "typical" (A. E. Hay, manuscript in preparation, 2008): the quadratic bottom friction coefficient $C_{D}=0.01$. We explore the importance of the magnitude of the friction coefficient, the choice of which is found to effect the flow significantly. On comparison with numerical model results and the field data, the analytic model is found to capture the dependence of the phase and amplitude responses of the oscillation on spatial position, and to changes in the friction coefficient.

[5] In section 2, the field site, observations and data analysis methods are discussed. In section 3, an analytical model is described. In 4 , the hydrodynamic model and 


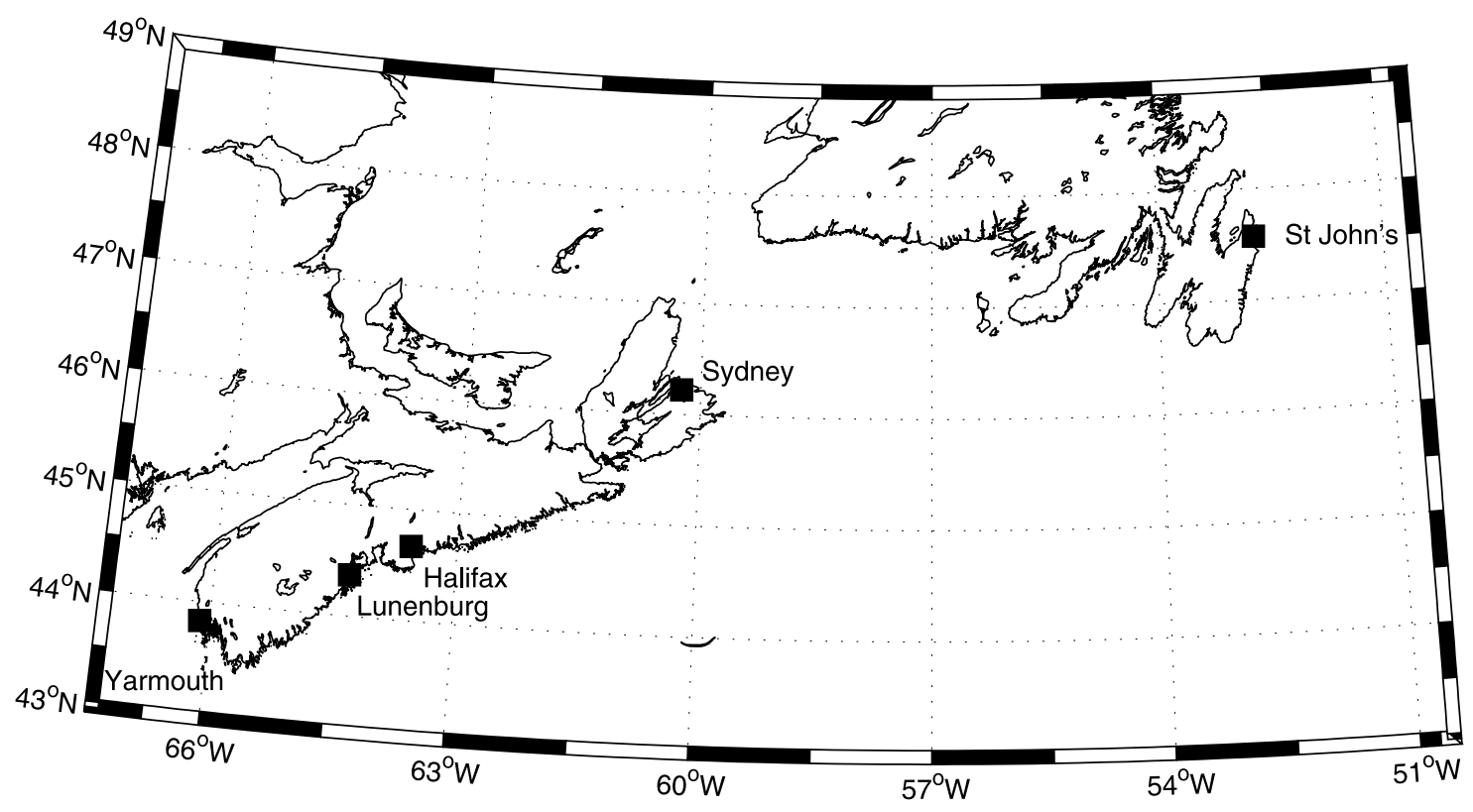

Figure 1. Atlantic coast of Canada showing the locations of Lunenburg Bay and the other harbors discussed in section 2.4 .

solution methods are introduced. Section 5 compares results from the three approaches and discusses the limitations of the analytical model. Conclusions are summarized in section 6 .

\section{Field Measurements}

\subsection{Instrument Locations}

[6] The bay bathymetry and locations of instruments deployed in 2005 are shown in Figure 2. The instrument array was focused within and close to Corkum's Channel. The array consisted of three RBR TDR2050 pressure and temperature sensors along the eastern side of the channel. Current profiles were measured by a $2 \mathrm{MHz}$ Nortek Aquadopp and a $1 \mathrm{MHz}$ Nortek AWAC deployed along the channel axis. A $600 \mathrm{kHz}$ RDI Acoustic Doppler Current Profiler (ADCP) was placed on the ebb tide jet axis within the main bay approximately $750 \mathrm{~m}$ outside of the channel mouth. The instrumentation at each observatory mooring consisted of a bottom-mounted Sontek Acoustic Doppler Profiler (ADP) and a Seabird PT39 pressure and temperature sensor.

[7] Additional pressure and velocity data from a separate experiment in September 2005 based around the Sculpin Shoal region near the mouth of the bay are included here to provide more complete spatial coverage. While these results are consistent with those reported from the above array, it is emphasized that the two data sets are not concurrent. Table 1 lists the instruments used in this study.

\subsection{Data Acquisition and Processing}

[8] The instruments in the channel and jet (4-9) recorded data over the period 15-30 August 2005, with the exception of the ADCP at 8 (Figure 2), which recorded data from 17-30 August 2005. The observatory instruments were not operational for the entire period 15-30 August, so the longest continuous data record (during the experiment period) was selected from these sensors. Data from the shoal experiment were from the period 1-15 September 2005. In order to obtain the longest possible data records for spectral analysis, values for occasional missing data points were obtained by linear interpolation. The instrument sampling protocols are listed in Table 1.

[9] The data were high-pass filtered using a Butterworth filter (fourth-order for RBR pressure-temperature sensors,

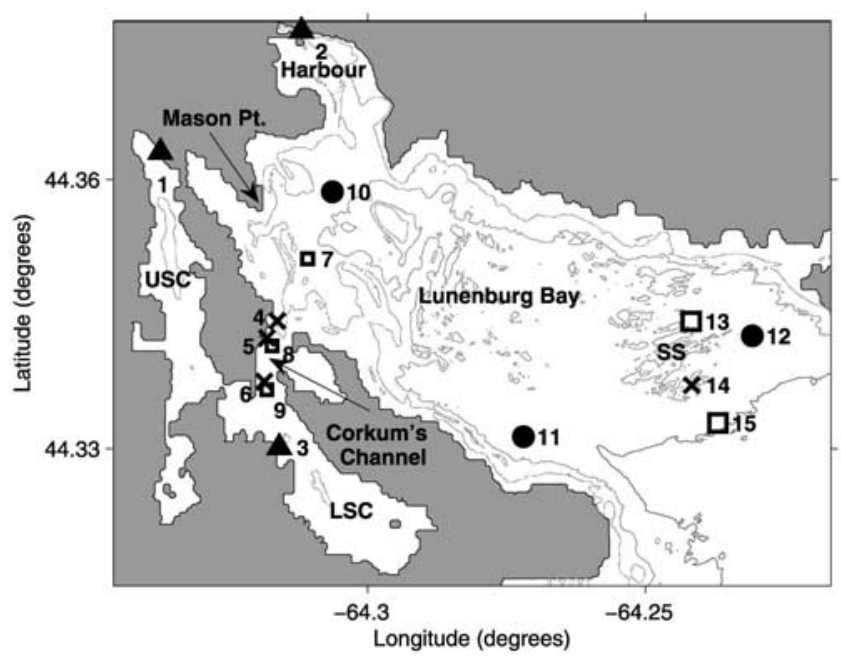

Figure 2. Bathymetry and instrument locations in Lunenburg Bay, Upper South Cove (USC) and Lower South Cove (LSC). The light, medium, and dark gray lines show the 5, 10 , and $20 \mathrm{~m}$ isobaths, respectively. The symbols represent observatory pressure and temperature sensors (closed triangles), observatory buoy nodes (black circles), the pressure and temperature sensors (multiplication signs), and acoustic Doppler profilers (squares) in the Corkum's Channel experiment in 2005. Instruments 13-15 were deployed as part of a separate experiment on Sculpin Shoal (SS) in September 2005. See Table 1 for further details. 
Table 1. Summary of the Instruments Deployed in Lunenburg Bay During the Summer of $2005^{\text {a }}$

\begin{tabular}{|c|c|c|c|c|c|c|c|}
\hline Position & Instrument & Make & Model & Frequency (MHz) & Cell Size $(\mathrm{m})$ & $\begin{array}{c}\text { Sampling } \\
\text { Frequency }(\mathrm{Hz})\end{array}$ & $\begin{array}{l}\text { Time Averaging } \\
\text { Window (min) }\end{array}$ \\
\hline $1^{\mathrm{b}}$ & PT sensor & Seabird & PT39 & - & - & 1 (on $10 / 15 \mathrm{~min})$ & 15 \\
\hline $2^{b}$ & PT sensor & Seabird & PT39 & - & - & 1 (on $11 / 15 \mathrm{~min})$ & 15 \\
\hline $3^{b}$ & PT sensor & Seabird & PT39 & - & - & 1 and 2 & 2 \\
\hline $4-6$ & PT sensor & RBR & TDR2050 & - & - & 1 & $1 / 60$ \\
\hline 7 & ADCP & RDI & Sentinel & 0.6 & 0.5 & 2 & 2 \\
\hline 8 & ADCP & Nortek & AWAC & 1 & 0.5 & 6.1 & 2 \\
\hline 9 & ADCP & Nortek & Aquadopp & 2 & 0.5 & 9 & 2 \\
\hline $10-12^{\mathrm{b}}$ & ADCP & Sontek & ADP & 1.5 & 0.5 & 0.1 (on $10 / 30 \mathrm{~min})$ & 30 \\
\hline $10-12^{b}$ & PT sensor & Seabird & PT39 & - & - & 0.67 (on $10 / 30 \mathrm{~min})$ & 30 \\
\hline $13^{\mathrm{c}}$ & ADCP & Nortek & Aquadopp & 2 & 0.5 & 14 (on $2 / 10 \mathrm{~min})$ & 10 \\
\hline $14^{\mathrm{c}}$ & PT sensor & RBR & TDR2050 & - & - & 1 & $1 / 60$ \\
\hline $15^{\mathrm{c}}$ & ADCP & Nortek & AWAC & 1 & 0.5 & $4.3($ on $2 / 10 \mathrm{~min})$ & 10 \\
\hline
\end{tabular}

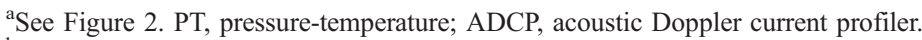

${ }^{\mathrm{b}}$ Includes instruments which form part of the coastal observatory.

${ }^{\mathrm{c}}$ Instruments deployed in September as part of a separate experiment.

fifth-order for all other instruments) with a cut-off frequency of $5 \mathrm{cpd}$. Time series of quantities which have been highpass filtered are denoted by a prime throughout this paper. The spectra were calculated using Hanning-windowed data segments with $70 \%$ overlap. The spectral parameters were chosen to give a frequency resolution of $\Delta f=0.0417 \mathrm{cph}$ for all instruments. All corresponding spectral densities have a minimum of 22 (pressure data) or 26 (velocity data) degrees of freedom, except for the ADCP (at site 7) and the ADP at site 12 (Figure 2). The pressure sensor on the ADCP malfunctioned after 5.5 days (resulting in only 12 degrees of freedom) and the ADP only had 4 days of continuous data, giving 6 degrees of freedom. However, the results shown were compared to results (from the same instrument) obtained using a longer data record from September and were consistent. Hence, we conclude that the results are representative of this period. The approximate $95 \%$ confidence intervals for the power spectral densities $S_{x x}(\omega)$ are given by

$$
\left[\frac{\nu S_{x x}(\omega)}{b_{\nu}(5)}, \frac{\nu S_{x x}(\omega)}{a_{\nu}(5)}\right]
$$
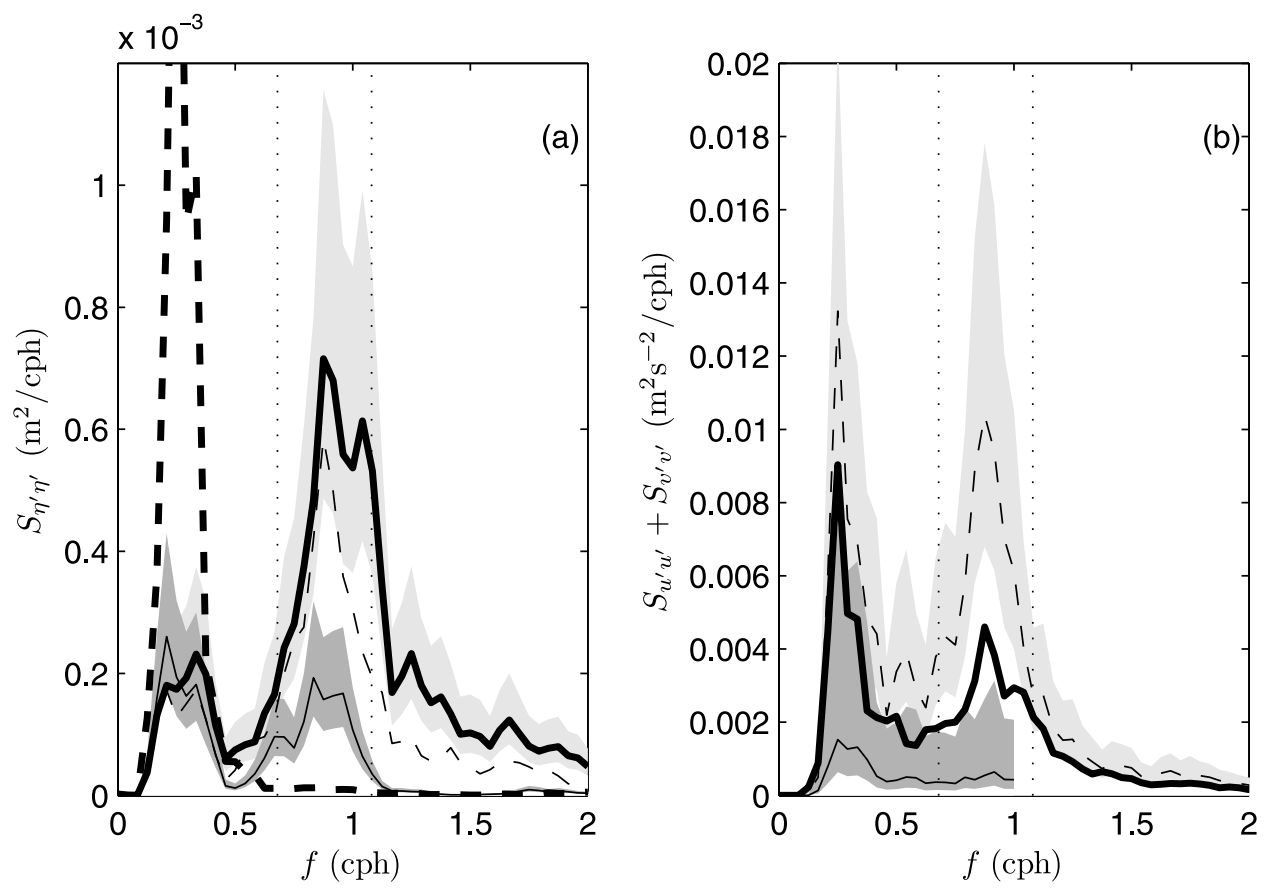

Figure 3. (a) Spectra of high-pass filtered pressure at selected locations. The solid thick line, the thin dashed line, and the thin solid line are the power spectral densities from instruments 2, 8, and 14, respectively (Figure 2). The thick dashed line is the spectrum from pressure sensor 1 in Upper South Cove. The 95\% confidence intervals are shown for instruments 2 and 14. (b) Spectra of high-pass filtered velocity. The solid thick line, the dashed line, and the thin solid line are the spectra from instruments 9,8 , and 12, respectively. The 95\% confidence intervals are shown for instruments 8 and 12 . In both Figures $3 \mathrm{a}$ and $3 \mathrm{~b}$ the vertical dotted lines show the frequency band over which the spectra have been integrated to give the variances used in Figure 4. 

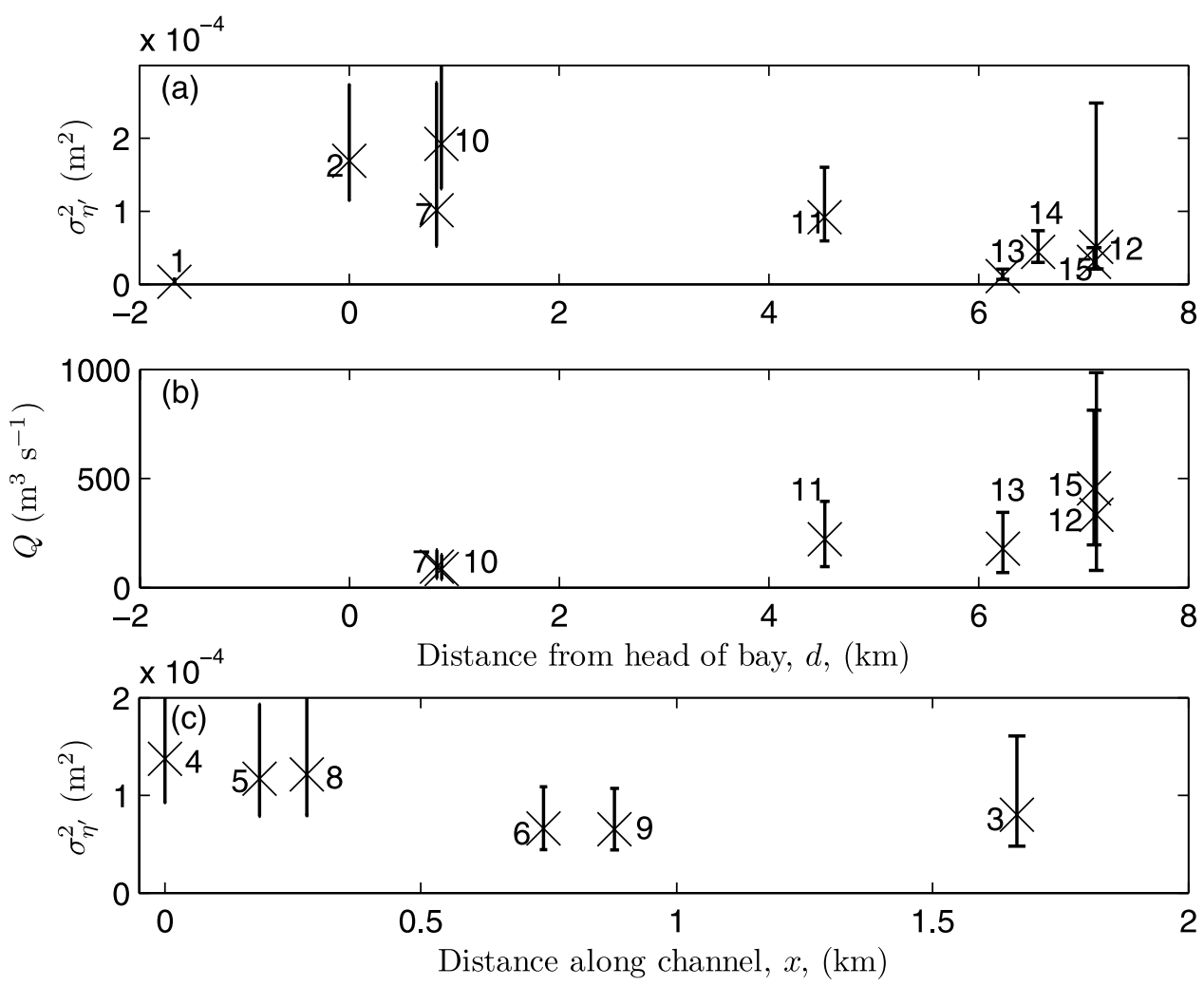

Figure 4. (a) Variances of the pressure spectra over the frequency band $0.68-1.08 \mathrm{cph}$ and (b) estimated RMS transport $Q$ as a function of position in the bay (see text for details). Similarly, (c) variances from the pressure spectra from instruments inside the channel and coves are plotted as a function of position along the channel. The numbers correspond to instrument positions (Figure 2). The error bars show the energy levels from the upper and lower $95 \%$ confidence intervals of the spectra.

where $v$ is the equivalent number of degrees of freedom and $a_{v}(5)$ and $b_{v}(5)$ are the 0.25 and $0.975 \%$ points of the $\chi^{2}$ distribution with $v$ equivalent degrees of freedom [Priestley, 1981, pp. 467-468].

\subsection{Pressure and Velocity Spectra}

[10] Representative power spectra for the high-pass filtered pressure data from selected instruments are presented in Figure 3a. The pressure spectra for all instruments (except site 3 in Upper South Cove) show a broadbanded peak centered at $f \approx 0.88 \mathrm{cph}$ or $T \approx 68 \mathrm{~min}$ and a peak at $f=0.25 \mathrm{cph}$ corresponding to the M6 tidal constituent. The thick solid line (instrument 2, close to the head of the bay) and dashed line (from instrument 8 in the channel) exhibit the highest peaks, while the peak in the $S_{\eta^{\prime} \eta^{\prime}}$ spectrum from the instrument close to the mouth of the bay (14) is smaller and broader. No peak around $0.88 \mathrm{cph}$ is present in the pressure spectrum from the sensor located in Upper South Cove, indicating that the higher frequencies were hydraulically choked at the narrow shallow entrance to this cove. The sum of the depth-averaged power spectra of the highpass filtered horizontal velocity components are presented in Figure 3b. Depth bins affected by surface interference were not included in this calculation. The spectra similarly reveal peaks at $f=0.25$ and $f=0.88 \mathrm{cph}$ and the energy levels around $f=0.88 \mathrm{cph}$ were 1 order of magnitude higher in the channel than elsewhere in the bay. We note that, for the spectrum from instrument 12 in Figure $3 \mathrm{~b}$ the Nyquist frequency is $1 \mathrm{cph}$, so there is unavoidable spectral aliasing for this instrument.

\subsection{Spatial Distribution of Elevation Variance and Volume Flux}

[11] The spectra were integrated to obtain the variances in a frequency band centered around $0.88 \mathrm{cph}(f=0.68-$ $1.08 \mathrm{cph})$. For instruments in the bay, the pressure variances are plotted in Figure $4 \mathrm{a}$ as a function of distance from the head of the bay. For the instruments in the channel and coves the variances are shown in Figure $4 \mathrm{c}$ as a function of the distance along the channel. The error bars indicate the upper and lower 95\% confidence intervals over the same frequency band. The distances were measured from Mason Point (Figure 2) and calculated as illustrated in Figure 5. For the pressure variances, the energy in the $\sim 1$-hour band decreases with distance from the head of the bay. This spatial distribution of energy in the pressure signal is consistent with a quarter-wave resonance in the main bay. An estimate of the period of this fundamental mode oscillation is given by

$$
T=(1+\epsilon)\left(4 \int_{0}^{L} \frac{\mathrm{d} x}{\sqrt{g h(x)}}\right)
$$

where $h(x)$ is the water depth at position $x$ and $\epsilon=0.31$ is a correction factor based on the aspect ratio (width/length) of 


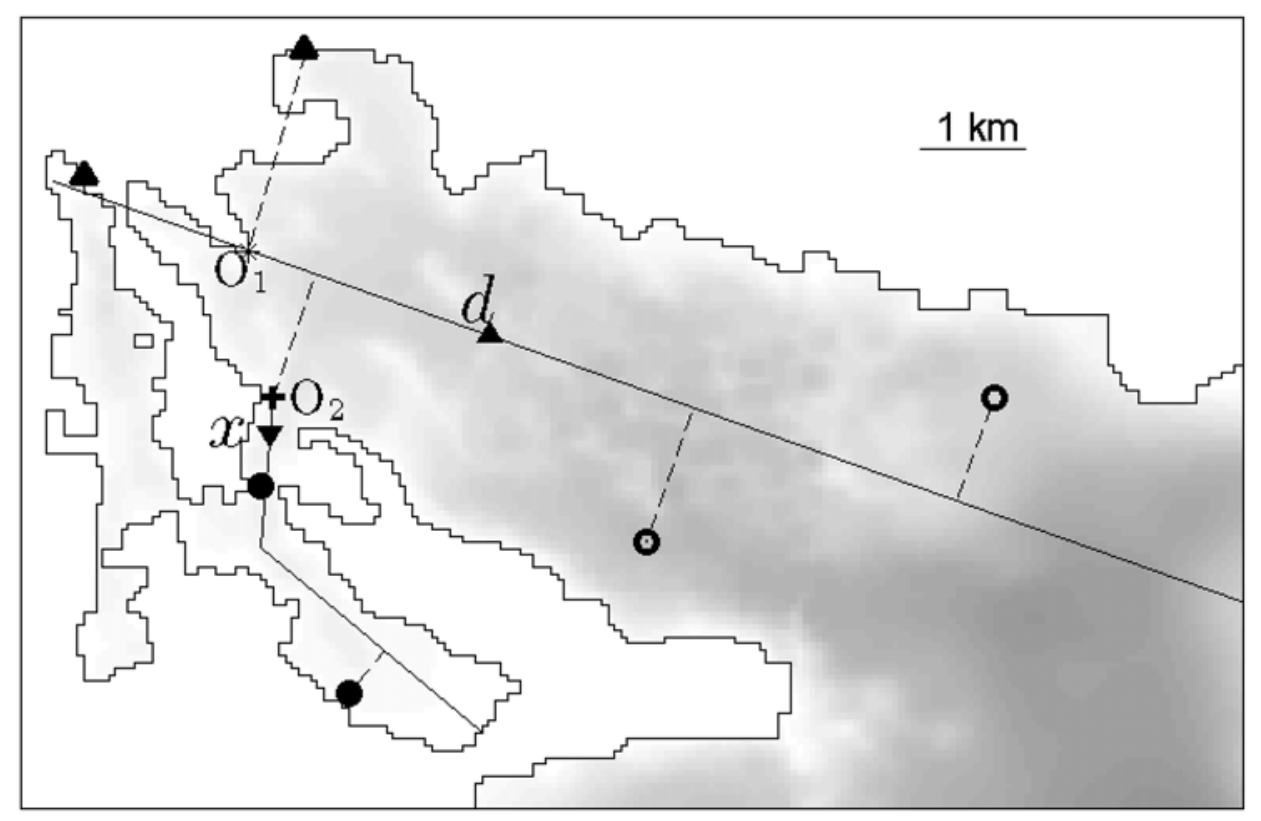

Figure 5. Schematic illustrating the axial distances of the indicate of instruments. For points inside the bay, the distance, $d$, is measured along the center axis from the origin $O_{1}$ to the point perpendicular to a given instrument. For instruments inside the channel and coves, the distance, $x$, is measured from the origin $\mathrm{O}_{2}$ along the center axis of the channel and cove. Note that for the sensor in Upper South Cove, $d<0$.

the bay [see Defant, 1961]. Using the depth averaged across the bay for $h(x)$ and integrating to the mouth of the bay (just inside of instrument 14 (Figure 2)), we obtain $T=67 \mathrm{~min}$ for an estimate of the quarter-wave resonance period, in excellent agreement with the observed period of $68 \mathrm{~min}$.

[12] The bathymetry of Lunenburg Bay is very irregular. Hence, for a quarter-wave resonance, it is also required that the volume flux in the resonance frequency band should increase with distance from the head of the bay, (rather than just the velocity variances at individual locations). Therefore, the velocity variances were roughly extrapolated to form an estimate of the average RMS transport over a half cycle of the oscillation $Q$ as

$$
Q=\frac{\sqrt{2}}{\pi} U_{R M S}^{\prime} \bar{h} W
$$

where $\bar{h}$ is the mean depth for a cross section of width W, and $U_{R M S}^{\prime}=\sqrt{\sigma_{u^{\prime}}^{2}+\sigma_{v^{\prime}}^{2}}$. The results for the instruments inside the bay are shown in Figure $4 \mathrm{~b}$ and we note that the evidence for such an increase of the RMS transport is inconclusive. However, the response peak at $0.88 \mathrm{cph}$ is significant and we use the numerical simulations (section 5) to examine the volume flux and verify the quarter-wave resonance a posteriori.

[13] Figure 6d shows daily variances and the associated $95 \%$ confidence intervals in the 1-hour band from the pressure sensor in Lunenburg Harbor throughout 2005. The time series were high-pass filtered with a cut-off frequency of $5 \mathrm{cpd}$ and then split up into segments two tidal cycles long ( $\sim 25$ hours). The ensemble-averaged spectrum for each data segment was calculated with a frequency resolution of $\Delta f=0.17 \mathrm{cph}$. The dotted lines mark the timing of the experiment. Similarly, the daily variances of sea surface elevation throughout 2005 were calculated from tide gauge data at all locations with permanent tide gauges on the open Atlantic coast of Nova Scotia and Newfoundland (Figure 1). These variances are also included in Figure 6. All of the tide gauges were sampled at $0.25 \mathrm{cph}$. In each case the data from the experimental period in August were used to identify the location of the highfrequency spectral peaks. The data were integrated in a frequency band of width $0.4 \mathrm{cph}$ around a selected peak with center frequency of $0.84 \mathrm{cph}$ for St. John's, $0.46 \mathrm{cph}$ for Sydney, $1.08 \mathrm{cph}$ for Halifax Harbor and $0.96 \mathrm{cph}$ for Yarmouth. The results indicate that oscillations existed in each harbor throughout the year. There is also a clear seasonal variability, a greater number of large-amplitude events occurring in the winter months. For example, during these events in Lunenburg, the amplitude of the oscillation of sea surface height reached up to $0.2 \mathrm{~m}$ in the harbor (a factor of 4 larger than during the experimental period). The Lunenburg results have also been compared with those from other years (2002 and 2004) and a similar seasonal variability was found.

[14] The forcing mechanisms for these oscillations are unclear and almost certainly vary between locations. However, there is some coherence between locations in the occurrence of the large amplitude events. Unsurprisingly, the correlation is highest between Halifax and Lunenburg, which are the least separated geographically. It is common for Fall storms to track northeastward along the coast [Eichler and Higgins, 2006; Mulligan et al., 2006] and these larger events are most likely related to the associated atmospheric forcing [de Jong et al., 2003; de Jong and 

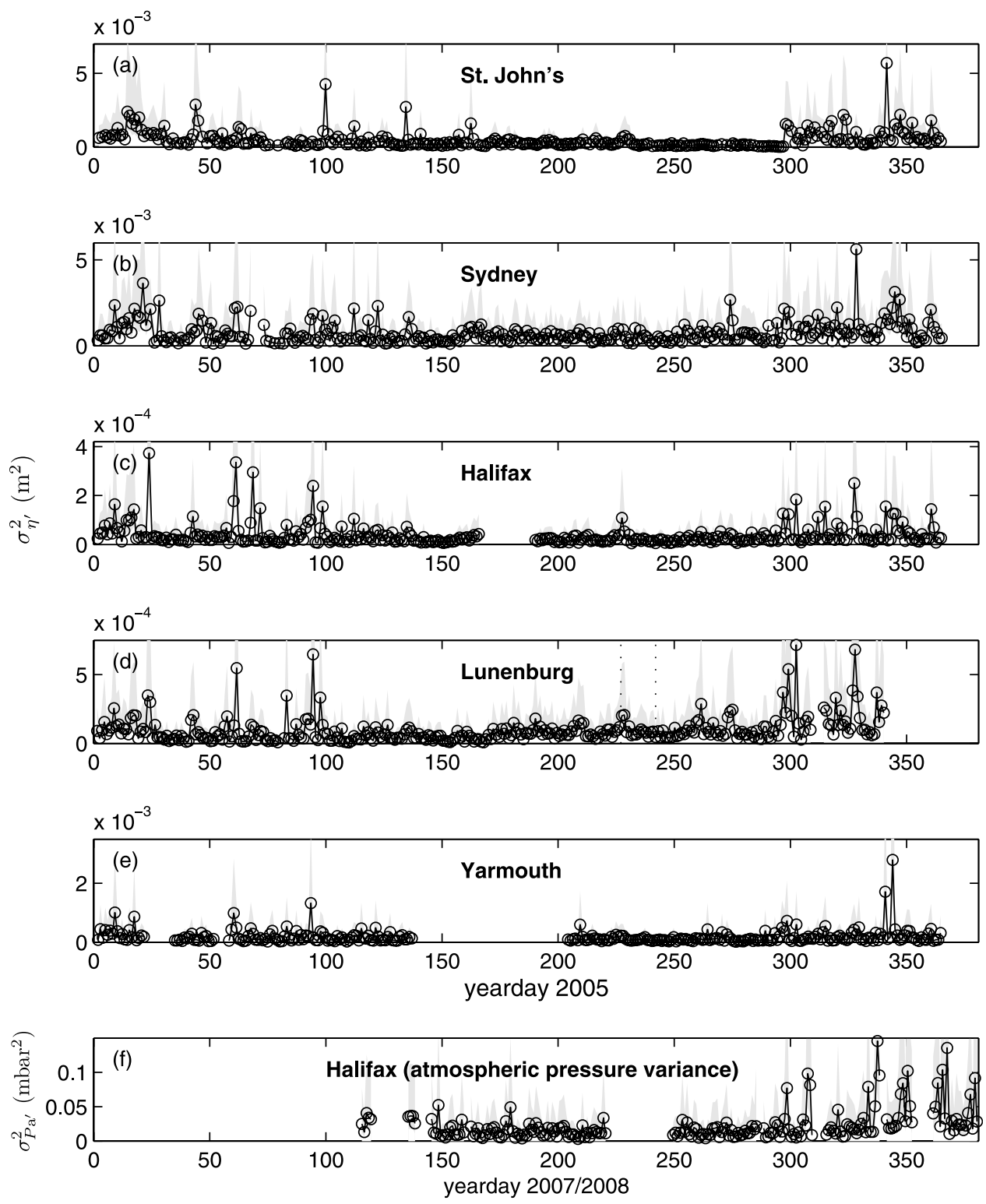

Figure 6. (a, b, c, d, and e) Year-long records of daily variances of high-pass filtered sea surface elevation data from harbors along the open coast of Atlantic Canada in 2005. For each location the spectra of highpass filtered sea surface elevation data were integrated over a frequency band of width $\Delta f=0.4 \mathrm{cph}$ centered on a high-frequency peak (near $1 \mathrm{cph}$ ). The shading shows the variances of the $95 \%$ confidence intervals. The dotted lines on the record from Lunenburg Harbor indicate the experimental period in August 2005. The locations are arranged from north to north in Figures 6a, 6b, 6c, 6d, and 6e. (f) Daily variances from high-pass filtered atmospheric pressure data from Halifax in 2007-2008.

Battjes, 2004]. High temporal resolution atmospheric data is not available for the region in 2005. However, the daily variances (over the frequency band $f=0-2 \mathrm{cph}$ ) of recently recorded (2007 and 2008) atmospheric pressure data at Dalhousie University in Halifax, Nova Scotia are shown in Figure 6f, and a similar seasonal variability to that in Figures $6 \mathrm{a}, 6 \mathrm{~b}, 6 \mathrm{c}, 6 \mathrm{~d}$, and $6 \mathrm{e}$ is observed. The atmospheric pressure data has a broadbanded spectrum at the higher frequencies (i.e., timescales of hours) tailing off around $2 \mathrm{cph}$. We suggest therefore that the oscillation in Lunenburg is an amplification of this energy at the intrinsic frequency of the bay.

\subsection{Amplification in the Tidal Channel}

[15] Time series of raw and high-pass filtered pressure data from a single day (21 August) from the ADCP in the center of the channel (Nortek AWAC at site 8 (Figure 2)) are shown in Figure 7 a. The $\sim 1$-hour oscillation is clearly seen in the filtered data despite a small sea surface displacement (sometimes observed to reach up to $\sim 0.08 \mathrm{~m}$ ), relative to the $\sim 1 \mathrm{~m}$ amplitude of the semidiurnal tide. Similar amplitudes were seen at the head of the bay and in the harbor. However, as noted previously, the energy levels of the velocity spectra in the $0.68-1.08$-cph frequency band were greatly amplified in the channel relative to the rest of 

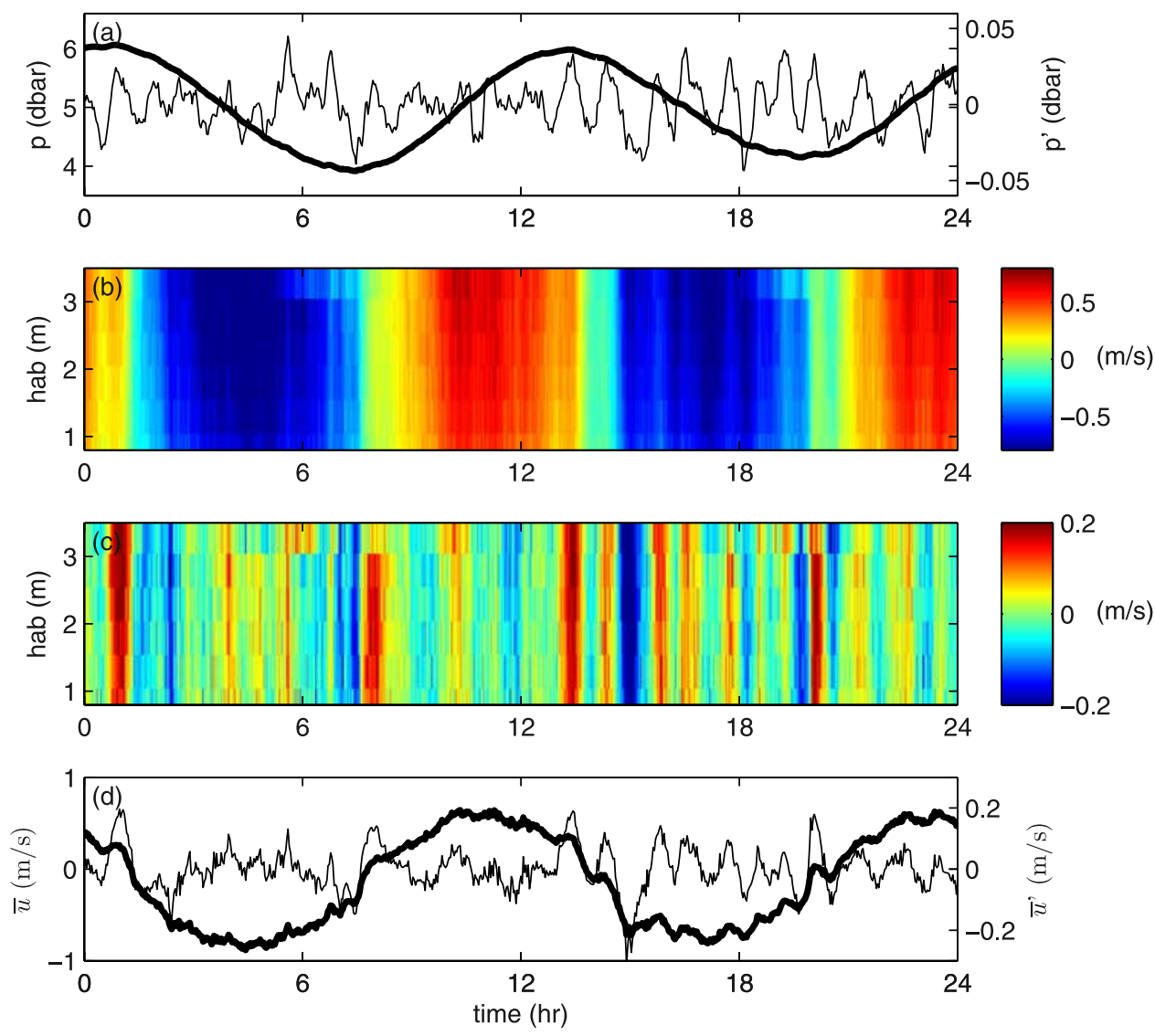

Figure 7. Velocity and pressure data from 21 August 2005 in the center of the channel (Nortek AWAC at 8 (Figure 2)). (a) Pressure both raw (thick) and the high-pass filtered pressure (thin). (b) Along-channel velocity. Positive values indicate flow into the coves (i.e., flood tide). (c) High-pass filtered along-channel velocity. (d) Depth-averaged along-channel velocities, both raw data (thick) and high-pass filtered (thin).

the bay (Figure 3b). In Figure 7 the velocities have been rotated into along- and across-channel components using a rotation angle based on a temporal mean of the depthaveraged flow direction. The axes are aligned such that $u$ is the along-channel velocity and is positive at flood tide (i.e., flow into the coves) and $v$ is the across-channel velocity with positive values for eastward flow. Figure $7 \mathrm{~b}$ shows the along-channel tidal velocities. The maximum along-channel velocity reached $\sim 1 \mathrm{~m} \mathrm{~s}^{-1}$, (while across-channel velocities were typically of order $\left.0.1 \mathrm{~m} \mathrm{~s}^{-1}\right)$. The high-pass filtered velocities in Figure 7c and the depth-averaged quantities in Figure $7 \mathrm{~d}$ reveal a pulsation in flow with amplitudes of around $0.2 \mathrm{~m} \mathrm{~s}^{-1}$ in the along-channel direction (and $0.05 \mathrm{~m}$ $\mathrm{s}^{-1}$ in the across-channel direction, not shown).

[16] The oscillation had a slight depth dependence with larger amplitudes closer to the surface. The amplitudes of the oscillation in the along-channel velocities reached up to $0.4 \mathrm{~m} \mathrm{~s}^{-1}$ on occasion (more frequently on the ebb), which represents a significant modulation (40\%) of the tidal velocities. Figure 8 shows pressure data (Figure 8a) and 4-hour windowed variances of the high-pass filtered pressure (Figure 8b) and along-channel velocity (Figure 8c) from the ADCP in the center of the channel for the entire experimental period. Figure 8 demonstrates that the oscillation was always present and that the motion is quasistationary over the 2-week timescale. However, there were occurrences of larger amplitude events indicating nonstationarity over a shorter timescale.

[17] An analytical model showing the quarter-wave resonance forcing a standing wave in the channel and pumping response (Helmholtz mode) in Lower South Cove is considered in the next section. Comparison of the analytical solutions with the field data and numerical results (section 5) demonstrates that the field data and numerical results are consistent with the presence of standing waves in the channel and coves.

\section{Analytical Model}

[18] Helmholtz oscillations often arise in basins attached to a large body of water through a channel or constriction. In coastal regions, they are typically generated by external oscillatory tides. The Helmholtz resonance is the lowest mode of response and involves a spatially uniform oscillation in sea surface elevation. Lateral motion is confined to a region near to the mouth of the basin. The mode has been called a pumping [Lee, 1971] or co-oscillating mode [Platzman, 1972] and it has been extensively studied [e.g., see Miles and Lee, 1975]. The response has also been examined in more complicated geometries, such as for a basin with sloping sidewalls [Green, 1992] or a sloping bottom [Maas, 1997]. However, for the present purposes, 

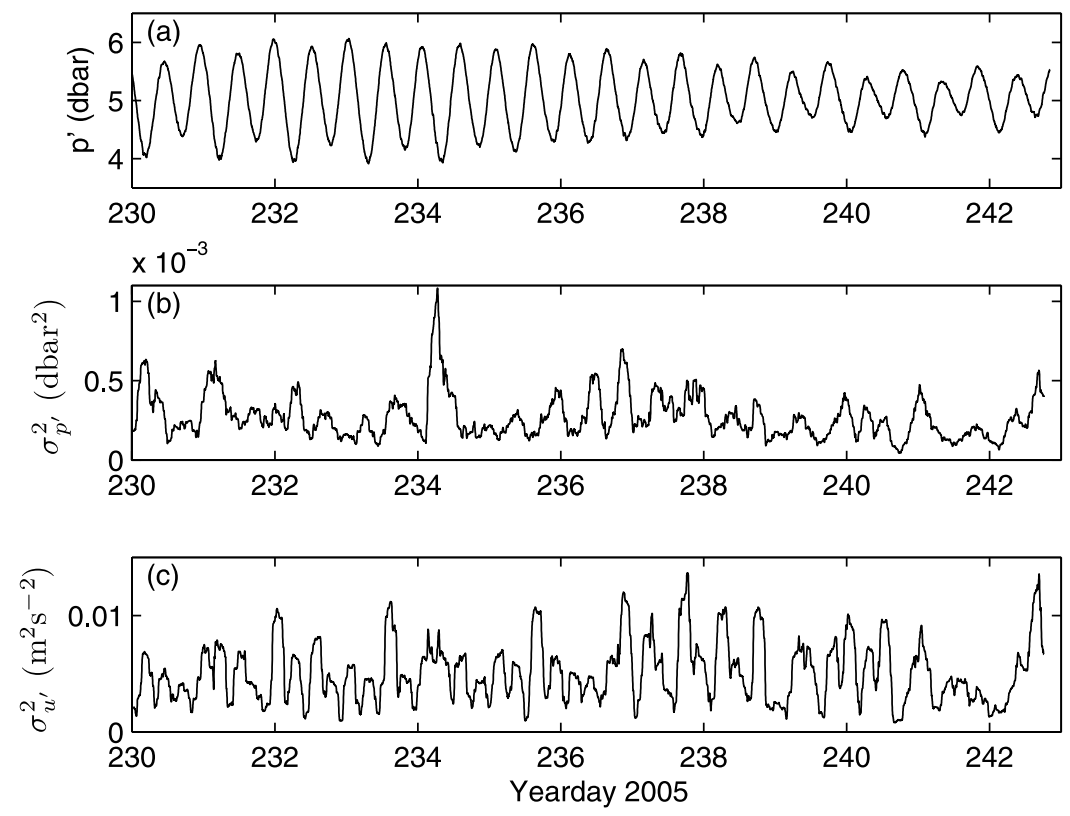

Figure 8. Velocity and pressure data over the experimental period from the center of the channel (Nortek AWAC at 8 (Figure 2)). (a) Pressure. (b) Windowed (4 hours) variance of high-pass filtered pressure. (c) Windowed (4 hours) variance of high-pass filtered along-channel velocity.

we adopt the standard picture of a Helmholtz mode in a uniform basin, as in the simplified geometry shown in Figure 9. The Helmholtz resonance frequency is given by

$$
\omega_{H}=\sqrt{\frac{W h g}{A L}}
$$

where $A$ is the basin area and $L, W$ and $h$ are the channel length, width and depth, respectively. The resonance period for Lower South Cove is poorly constrained. Uncertainties arise in the definition of the location of the end of the channel or start of the Cove (particularly given the channel curves), the channel width (whether or not to include the quiescent regions of almost no flow at the sides) and the depth is not completely uniform. Nonetheless, we can estimate $\bar{h}=5 \mathrm{~m}, L=1-2 \mathrm{~km}, W=200-400 \mathrm{~m}$ and $A=2-$ $3 \times 10^{6} \mathrm{~m}^{2}$, which gives $T_{H}=60 \pm 20 \mathrm{~min}$ for the Helmholtz period. Importantly, the Helmholtz frequency and the friction term depend on the water depth $\bar{h}+\eta_{M 2}(t)$ and tidal velocity $U_{M 2}$, which vary throughout the tidal cycle. The base Helmholtz period $T_{H 0}$ is modified as

$$
T_{H}(t)=T_{H 0} \sqrt{\frac{\bar{h}}{\bar{h}+\eta_{M 2}(t)}} .
$$

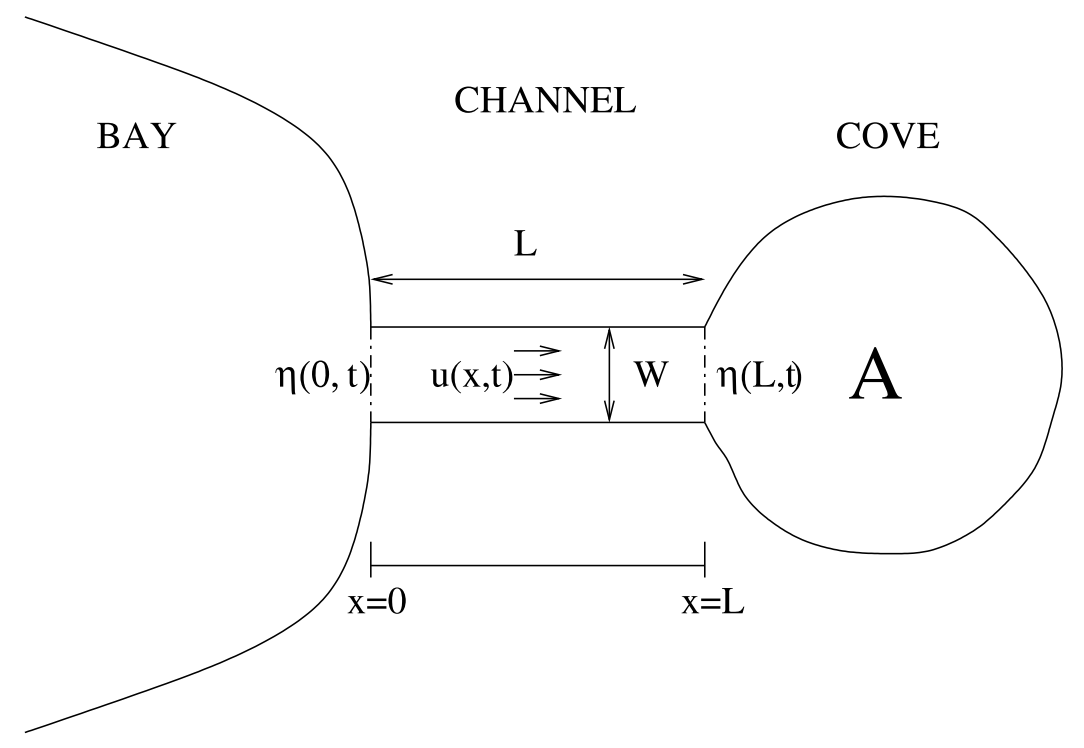

Figure 9. Schematic domain for the analytic model. The depth of the channel is $h(x, t)$. 
This dependence on the phase of M2 tide has been included (as cos and sin terms for $U_{M 2}$ and $\eta_{M 2}$, respectively) in later calculations of amplitudes and phases. We note also that friction acts to decrease the Helmholtz frequency, so the actual Helmholtz period will be increased slightly above the values in (5).

[19] Position along the channel is given by $x$, with the origin at the channel entrance and $x=L$ at the entrance to the cove. Sea surface elevation is denoted by $\eta$, and alongchannel velocity by $u$. The flow within the channel is forced by an oscillatory variation in sea level at the channel entrance. The response in the cove is assumed to be a pure Helmholtz mode, i.e., the sea surface elevation executes a spatially uniform oscillation. Conservation of mass ensures

$$
\frac{\partial \eta}{\partial t}=-h \frac{\partial u}{\partial x}
$$

and the momentum equation, ignoring the inertial acceleration terms, is

$$
\frac{\partial u}{\partial t}=-g \frac{\partial \eta}{\partial x}-\frac{\tau}{\rho_{0} h}
$$

where $\tau$ is the bottom stress given by,

$$
\frac{\tau}{\rho_{0}}=r u h,
$$

with

$$
r=\frac{C_{D}|\mathbf{U}|}{h},
$$

where $C_{D}$ is the drag coefficient for quadratic friction and $\mathbf{U}$ is the total velocity (tidal plus oscillation) along the channel. However, we approximate $|\mathbf{U}|$ as the tidal velocity $\left|U_{T}\right|$ to keep the model linear. We observe that in the model the tidal velocity goes through zero at slack tide and hence friction also reduces to zero. This zero velocity is physically unrealistic so we add a small RMS "noise velocity" $u_{n}=$ $0.04 \mathrm{~m} / \mathrm{s}$ (consistent with the velocity spectra from the instrument in the center of the channel), to give

$$
r=\frac{C_{D}\left(\left|U_{T}\right|+u_{n}\right)}{h} .
$$

The resistance factor is assumed to vary sufficiently slowly over the 1-hour timescale that it can be taken as constant throughout the derivation of the solutions which follows. Hence, differentiating (6) with respect to $x$ and (7) with respect to $t$ and combing, yields

$$
\frac{\partial^{2} u}{\partial t^{2}}+r \frac{\partial u}{\partial t}=g h \frac{\partial^{2} u}{\partial x^{2}}
$$

The boundary condition at the channel mouth $(x=0)$ is

$$
\eta=a_{f} \exp \left(i \omega_{f} t\right),
$$

where $a_{f}$ and $\omega_{f}$ are the forcing amplitude and frequency (in $\mathrm{rad} / \mathrm{s}, \omega=2 \pi f$ for $f$ in $\mathrm{Hz}$ ), respectively, i.e., in the present case, the amplitude and frequency of the quarter-wave oscillation at the channel entrance. Both $a_{f}$ and $\omega_{f}$ are real. The boundary condition at the end of the channel and entrance to the cove $(x=L)$ is that for any pumping mode (including a Helmholtz resonance), i.e.,

$$
\frac{\partial \eta}{\partial t}=\frac{h W}{A} u(L, t) .
$$

The governing equation (11) is satisfied by a wave solution of the form

$$
u(x, t)=\left(\alpha e^{i k x}+\beta e^{-i k x}\right) e^{i \omega_{f} t},
$$

where $\alpha$ and $\beta$ are constants and $k$ is the wavenumber. Substituting into (11) gives

$$
k^{2}=\frac{\omega_{f}}{g h}\left(\omega_{f}-i r\right)
$$

and therefore

$$
k=\frac{\omega_{f}}{\sqrt{g h}}\left(1-\frac{i r}{\omega_{f}}\right)^{1 / 2} .
$$

Noting that $r / \omega_{f} \ll 1$, we expand (16) as

$$
k \approx \frac{\omega_{f}}{\sqrt{g h}}\left(1-\frac{i r}{2 \omega_{f}}\right)
$$

i.e.,

$$
k \approx k_{0}-i \gamma,
$$

where

$$
k_{0}=\frac{\omega_{f}}{\sqrt{g h}} \quad \text { and } \quad \gamma=\frac{r}{2 \sqrt{g h}} .
$$

The wave decay lengthscale is given by $1 / \gamma$ and is around $40 \mathrm{~km}$ for a drag coefficient of $C_{D}=0.0023$. Therefore, the solution (14) is a standing wave pattern composed of two weakly decaying waves propagating in opposite directions. Applying equation (6) and the boundary condition equation (12) yields

$$
\beta=\frac{\omega_{f} a_{f}}{k h}+\alpha
$$

and using equation (13)

$$
\alpha=\frac{1}{2 \cos k L}\left(\frac{i \omega_{f} A \hat{a}}{h W}-\frac{\omega_{f} a_{f} e^{-i k L}}{k h}\right),
$$

where $\hat{a}$ is the surface elevation amplitude inside the cove $(x \geq L)$. Upon substitution of the Helmholtz frequency $\omega_{H}^{2}=W h g / A L$ and after some algebraic manipulation, this amplitude is given by

$$
\hat{a}=\frac{a_{f}}{\cos k L}\left[\frac{k L \omega_{H}^{2}}{k L \omega_{H}^{2}-\left(\omega_{f}^{2}-i r \omega_{f}\right) \tan k L}\right] .
$$


An alternative and elegant derivation of equation (22) above, was brought to our attention by Professor J.A. Battjes in his review of the manuscript and follows Dronkers [1964] (by writing the linearized equations for flow in a prismatic channel in terms of the surface elevation $\eta$ and discharge).

[20] Hence, the final solutions for $\eta(x, t)$ and $u(x, t)$ are

$$
\eta(x, t)=a(x) e^{i \omega_{f} t}=a_{f}\left\{e^{-i k x}+F\left(k L, \omega_{H}, \omega_{f}\right) \frac{\sin k x}{\cos k L}\right\} e^{i \omega_{f} t}
$$

and

$$
u(x, t)=\frac{a_{f} \omega_{f}}{k h}\left\{e^{-i k x}+i F\left(k L, \omega_{H}, \omega_{f}\right) \frac{\cos k x}{\cos k L}\right\} e^{i \omega_{f} t},
$$

where

$$
F\left(k L, \omega_{H}, \omega_{f}\right)=\left[\frac{\omega_{f}^{2}-i r \omega_{f}}{k L \omega_{H}^{2} \cos k L-\left(\omega_{f}^{2}-i r \omega_{f}\right) \sin k L}+i e^{-i k L}\right] .
$$

We define the dimensionless magnitude and the phase of the sea surface oscillation along the channel (relative to the channel entrance) as

$$
G_{\eta}(x)=\frac{|a(x)|}{a_{f}}
$$

and

$$
\phi_{\eta}(x)=\tan ^{-1}\left(\frac{\Im[a(x)]}{\Re[a(x)]}\right) .
$$

For a channel of depth $h=5 \mathrm{~m}$, the shallow water wave speed is $c \approx 7 \mathrm{~m} \mathrm{~s}^{-1}$. Therefore, a forcing period of $68 \mathrm{~min}$ results in a wavelength of $28.5 \mathrm{~km}$, more than 10 times longer than the channel length of $2 \mathrm{~km}$. Hence, a small $k L$ approximation can be made and the following expression for $\hat{a}$ is obtained

$$
\hat{a} \approx \frac{a_{f} \omega_{H}^{2}}{\omega_{H}^{2}-\omega_{f}^{2}+i r \omega_{f}}=\frac{a_{f}\left[\omega_{H}^{2}\left(\omega_{H}^{2}-\omega_{f}^{2}\right)-i r \omega_{f} \omega_{H}^{2}\right]}{\left(\omega_{H}^{2}-\omega_{f}^{2}\right)^{2}+r^{2} \omega_{f}^{2}},
$$

which is the solution to the simplified forced (Helmholtz) oscillator problem with constant $u$, and $\eta$ a linear function of $x$. Furthermore, the phase is seen to depend on the relative magnitudes of the forcing and Helmholtz frequencies. The imaginary part of (28) is always negative. If $\omega_{H}<\omega_{f}$, then the real part of (28) is positive so the phases at $x=L$ must lie between 0 and $-90^{\circ}$, whereas if $\omega_{H}<\omega_{f}$, the real part is negative and the phases will fall between $-180^{\circ}$ and $-90^{\circ}$.
[21] It is also instructive to consider the small $k L$ approximation for $a(x)$ :

$$
\begin{aligned}
& a(x)=a_{f} \\
& {\left[\frac{\left(\omega_{H}^{2}-\omega_{f}^{2}\right)^{2}+r^{2} \omega_{f}^{2}+\omega_{f}^{2}\left(\omega_{H}^{2}-\omega_{f}^{2}-r^{2}\right) x / L-i r \omega_{f} \omega_{H}^{2} x / L}{\left(\omega_{H}^{2}-\omega_{f}^{2}\right)^{2}+r^{2} \omega_{f}^{2}}\right] .}
\end{aligned}
$$

The third term in the numerator demonstrates that the oscillation amplitude also depends on the relative magnitudes of the forcing and Helmholtz frequencies $\left(\omega_{f}\right.$ and $\left.\omega_{H}\right)$. If $\omega_{H}<\omega_{f}$, this term provides the single negative contribution to $|a(x)|$ and reduces both the amplitude and the rate of change of amplitude along the channel compared to the case in which $\omega_{H}>\omega_{f}$. However, the expression (29) indicates that the value of $r$ and hence $C_{D}$ will have a large effect on the amplitude along the channel. For $C_{D}=0.0023$, it is found that the squared contribution from the fourth term dominates the numerator, while for $C_{D}=0.01$, this contribution is balanced by the large negative term (for $\omega_{H}<\omega_{f}$ and $\omega_{H} \approx \omega_{f}$ ). Additionally, for $C_{D}=0.01$ the denominator of (29) is 1 order of magnitude larger than for $C_{D}=0.0023$. This dependence on friction is indeed clearly seen in the solutions plotted in Figure 10. The solutions are for a case with $\omega_{H}<\omega_{f},\left(\omega_{H}=1.34 \times 10^{-3} \mathrm{rad} \mathrm{s}^{-1}\right.$ and $\omega_{f}=$ $1.54 \times 10^{-3} \mathrm{rad} \mathrm{s}^{-1}$, or $T_{H}=78 \mathrm{~min}$ and $\left.T_{f}=68 \mathrm{~min}\right)$. The solutions using $C_{D}=0.0023$ are shown by the thin lines, while the solutions using $C_{D}=0.01$ (thick lines) show that the amplitude at the end of the channel is reduced by a factor of 2 relative to the low-friction case. The phases in the two solutions differ by $33^{\circ}$ at the end of the channel and up to $66^{\circ}$ at $x / L=0.48$. Further results are plotted in section 5 for comparison with numerical results and observations (Figures 11, 12, 13, and 14). The variation of the resistance factor over the tidal cycle has been taken into account as shown in equation 10 in the calculation of all results shown in Figures 10, 15, 16, 17, 18, and 19. Figures 17 and 18 are analytical solutions showing the modulation of the 1-hour oscillation by the tidal flow, whereas Figures 10, 15, 16, and 19 show tidally averaged quantities, which are denoted by an overbar throughout the manuscript.

\section{Numerical Modeling}

[22] Numerical simulations were conducted using the hydrodynamic package Delft3D in depth-averaged barotropic mode. The simulations aimed to examine whether the simple physics in the well-constrained analytical model were sufficient to understand predictions from the numerical model, which includes the nonlinear terms and more realistic geometry and bathymetry. In particular, we aimed to determine whether the simulations gave closer agreement to the field data than the analytical model. Moreover, the simulations allowed investigation of the response of the system to changes in friction coefficient and forcing frequency. The Delft3D-FLOW module solves the unsteady shallow water equations for an incompressible fluid. The equations were discretized on a staggered (Arakawa-C) grid. For details on numerical aspects of the model and its 

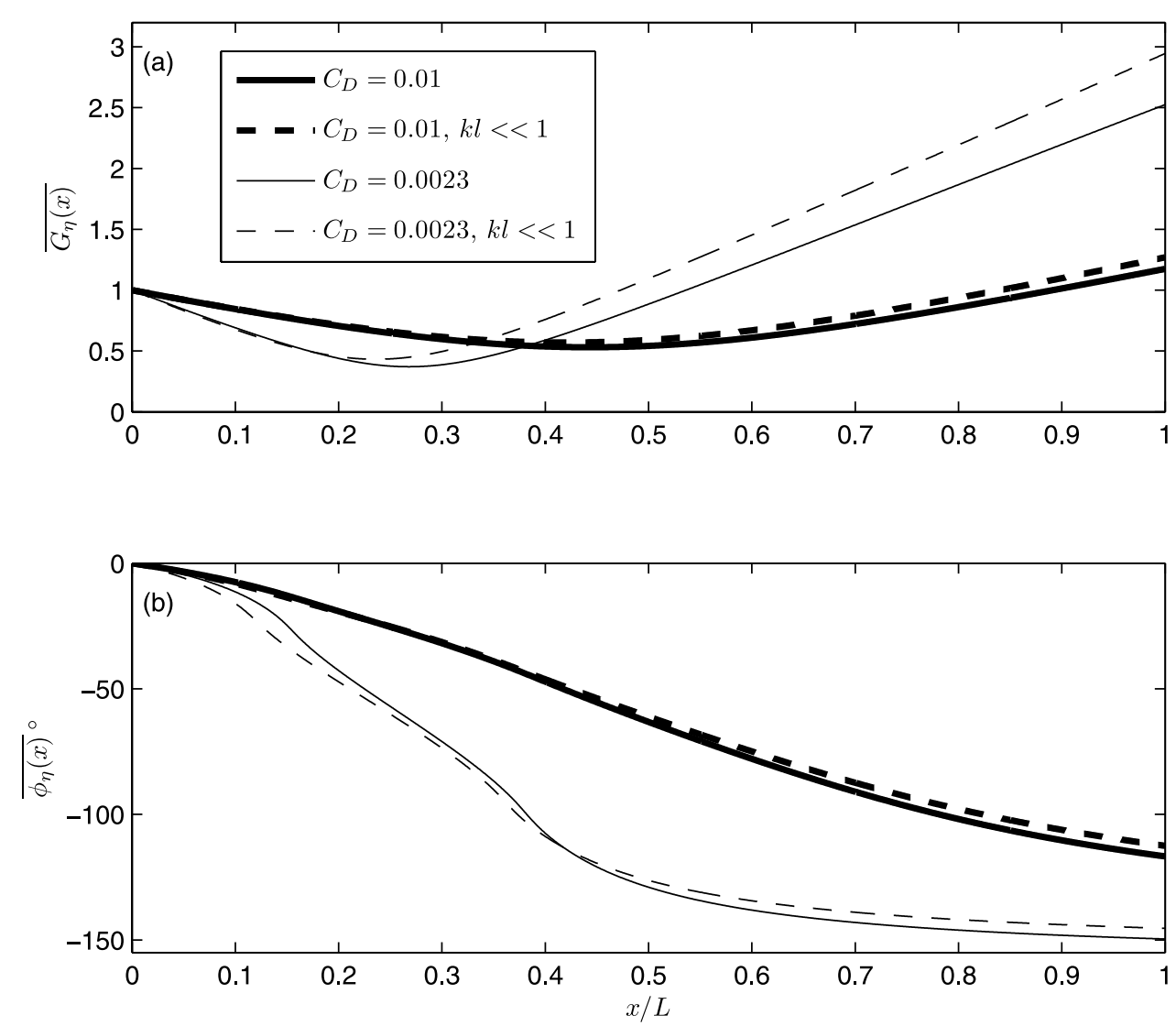

Figure 10. (a) Dimensionless magnitude and (b) phase of sea surface elevation along the channel (relative to the channel entrance) as given by (26) and (27). The solutions are means over the M2 tidal cycle. Thick lines indicate $C_{D}=0.01$, thin lines indicate $C_{D}=0.0023$, and dashed lines indicate the small $k L$ approximation. $T_{H}=78 \mathrm{~min}, T_{f}=68 \mathrm{~min}$, and $L=2000 \mathrm{~m}$.

extensive validation see, for example, papers by Elias et al. [2000] and Lesser et al. [2004].

\subsection{Model Configuration}

[23] We consider a simplified model in which flow is forced only at the open boundary of the bay. We force at the frequency of the M2 tidal constituent. As the forcing for the quarter-wave oscillation most likely originates from outside the bay, we also apply a forcing component for a range of oscillation frequencies at the open boundary. No atmospheric forcing is applied.

[24] Zero normal velocity and free slip (no shear stress) conditions are applied at the closed boundaries. At the open boundary the flow is forced with a harmonic Riemann boundary condition. This condition is based on a linearized Riemann invariant, $F_{R}=U+\eta \sqrt{\frac{g}{h}}$, (units $\mathrm{m} \mathrm{s}^{-1}$ ). The condition is nonreflective for outgoing waves normal to the boundary and reduces reflections for obliquely incident waves. Thus, $F(t)$ is specified on the boundary, where

$$
F(t)=\sum_{i} F_{R i} \cos \left(\omega_{i} t-\phi_{i}\right)
$$

in which $\omega_{i}$ and $\phi_{i}$ are the frequency and phase of component $i$.

\subsection{Solution Methods}

[25] A uniform grid with resolution of $60 \mathrm{~m}$ was implemented within Lunenburg Bay and an idealized outer domain (Figure 11). The large grid was used to ensure that the frequency of any numerical oscillations (or eigenmodes) of the domain was distinct from the forcing frequency. Output variables at the observation points (including the locations of the instruments) were written to file every $2 \mathrm{~min}$. The simulations were started from rest with uniform sea surface displacement $\eta$ throughout the domain and evolved to a cyclicly steady state. The time step was $15 \mathrm{~s}$. The amplitude of the initial transient was reduced by using a smoothing time of $600 \mathrm{~s}$, during which the boundary forcing was gradually applied. A horizontal eddy viscosity of $v_{H}=$ $1 \mathrm{~m}^{2} \mathrm{~s}^{-1}$ was used. Forcing at two frequencies was applied through the Riemann boundary conditions: the M2 tidal frequency (with amplitude $F_{R}=0.35 \mathrm{~m} \mathrm{~s}^{-1}$ ); and one other "oscillation" frequency (with amplitude $F_{R}=0.005 \mathrm{~m} \mathrm{~s}^{-1}$ ). The values of the Riemann invariants $F_{R}$ were chosen to give flow conditions, which were representative of the field conditions (especially those in the channel) during the experiments. That the model performance does, in essence, reproduce the field conditions is later demonstrated in Figures 12 and 13 and the following section. 


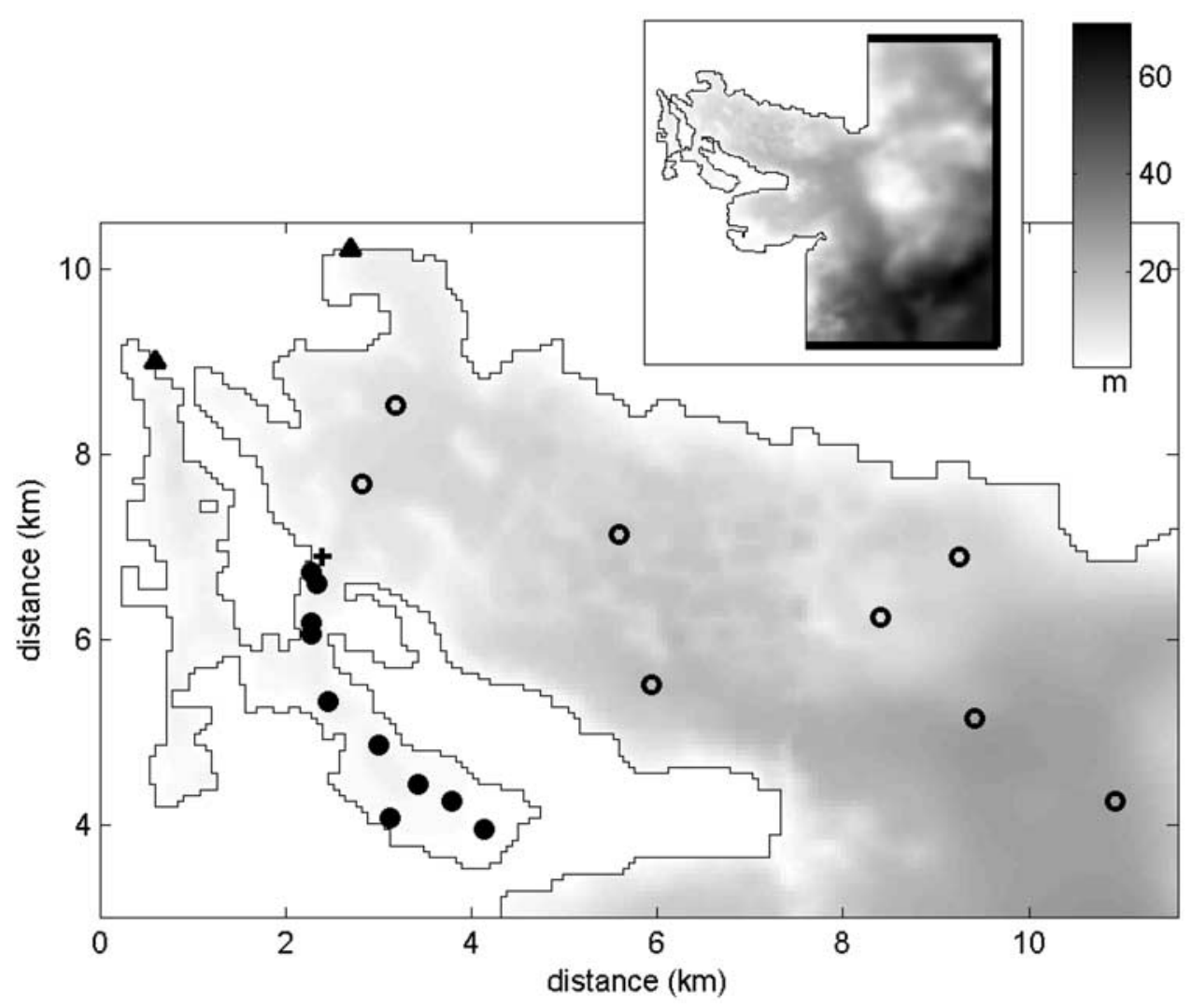

Figure 11. (top) Numerical domain and bathymetry showing Lunenburg Bay connected to an idealized outer domain. Forcing was applied at the outer north, east, and north boundaries, indicated by the thick black lines. (bottom) The location of measurement points (instrument locations and selected others) for the phase difference calculations (see section 5.1). Phase differences were calculated relative to the pressure sensor at the channel entrance (addition signs). The remaining symbols indicate points in the main bay (open circles), instruments in the channel and Lower South Cove (closed circles), and pressure sensors in the harbor and Upper South Cove (closed triangles).

[26] Although the Helmholtz period cannot be determined precisely from the bathymetry, with the numerical simulations it is possible to examine the effect of the relative magnitudes of $T_{H}$ and $T_{f}$ (as predicted by the analytical model to be important) by varying instead the period of the forcing. If we assume the maximum amplitude response of the oscillation occurs when the forcing frequency is close to the Helmholtz frequency, then these runs also allow for estimation of the actual Helmholtz period. Hence, a set of runs was conducted in which the oscillation frequency ranged from $f=0.58 \mathrm{cph}$ to $f=1.38 \mathrm{cph}$ and included the observed quarter-wave resonance frequency $(f=0.88 \mathrm{cph})$. For some simulations a uniform bottom friction coefficient was used, $C_{D}=2.3 \times 10^{-3}$. Other runs were conducted using a variable bottom friction coefficient. Although no measurements of the bottom friction coefficient have been made inside the coves, we hypothesize that its value will be closer to that inside the channel than the value in the bay, owing to the similarity of bottom type and water depth between the channel and coves. Therefore, for some runs the measured (in the channel) high value of $C_{D}=0.01$ was used inside the channel and coves, while in others a lowvalue $C_{D}=6.1 \times 10^{-5}$ was also used. In the main region of the bay, the coefficient remained at $C_{D}=2.3 \times 10^{-3}$. A roughly semicircular region of radius $700 \mathrm{~m}$ at the channel mouth was used to smooth $C_{D}$ between the two sections.

\section{Results}

[27] The model was run for several tidal cycles and all of the results shown here are from the periodically steady state. We note that the aim of the present simulations is not to reproduce observed flows in exact detail but rather to examine the interaction of a small amplitude oscillation with the tidally forced flows and Helmholtz resonance. Despite the relatively simple boundary conditions, the simulations capture the dominant large-scale flow features (Figures 12a and 12b): the pronounced jet on ebb tide, the asymmetry of flow between the ebb and flood phases of the tidal cycle and the choking of the flows by the constriction into Upper South Cove (Figure 2). These results are in reasonable agreement with other more detailed studies of the bay [Sturley and Bowen, 1996; Sheng and Wang, 2004].

[28] The time series of predicted sea surface elevation from the model reveals an M2 tidal amplitude at the entrance to the channel (instrument 4 (Figure 2)) of around $0.7 \mathrm{~m}$, close to the observed values for the experimental period (Figure 13a). Similarly, the high-pass filtered amplitudes of sea surface elevation (dominated by the oscillation 

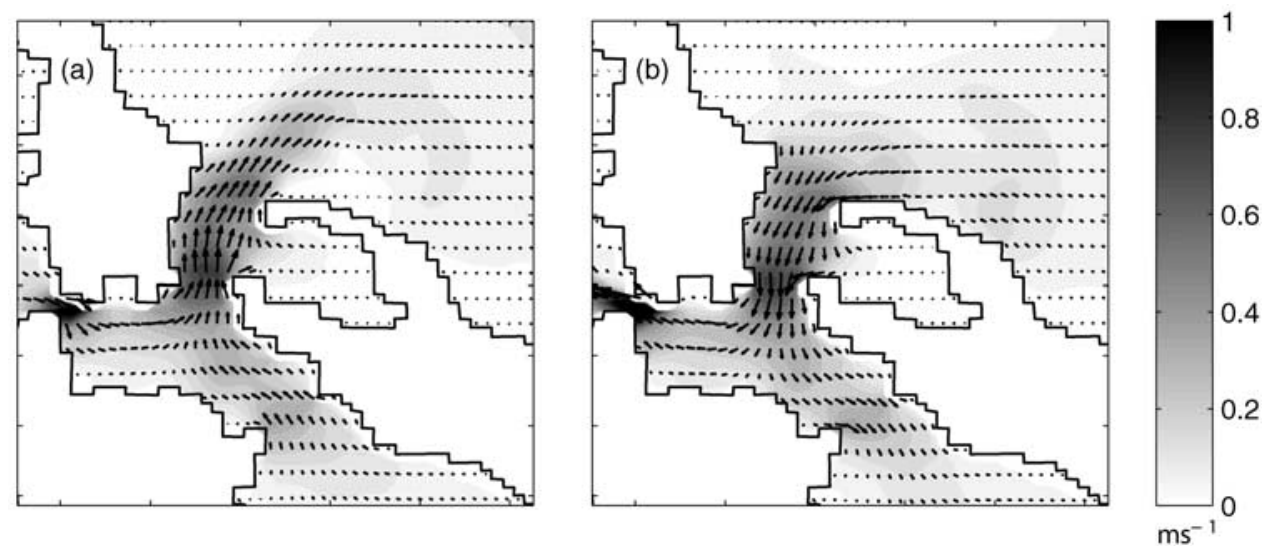

Figure 12. Depth-averaged velocities from the numerical model during (a) maximum ebb and (b) maximum flood. $C_{D}=0.01$ in the channel and coves, and $T_{f}=68 \mathrm{~min}$.

in the $\sim 1$-hour band) between the model and field data are in good agreement, with maximum values of around $0.1 \mathrm{~m}$ and typical values of around $0.06 \mathrm{~m}$, although there is considerably more variability in the field data (Figure 13b). Inside the channel (instrument 8 (Figure 2)), maximum predicted northward velocities were around $0.4 \mathrm{~m} \mathrm{~s}^{-1}$, which matched the lower end of the range observed values (Figure 13c). However, as in the field observations, the predicted M2 tidal velocities were substantially modulated (by $0.2 \mathrm{~m} / \mathrm{s}$, or $45 \%$ ) at the oscillation frequency (Figure 13d).
[29] Figure 14 illustrates the spatial distributions of RMS sea surface elevation and RMS velocity in response to the forcing at $0.88 \mathrm{cph}$ from a run with the higher bottom friction coefficient. Pressure and velocity fields from the numerical runs stored at 10-min intervals were high-pass filtered. The RMS sea surface elevation $\sigma_{\eta^{\prime}}$ in the frequency band $f=0.68-1.08 \mathrm{cph}$ is given in Figure 14a. The numerical results are consistent with the sparser observational data (Figure 4a): that is, inside the bay, RMS sea surface elevation decreases toward the mouth of the bay. As in the field data, $\sigma_{\eta^{\prime}}$ levels in Upper South Cove are low.
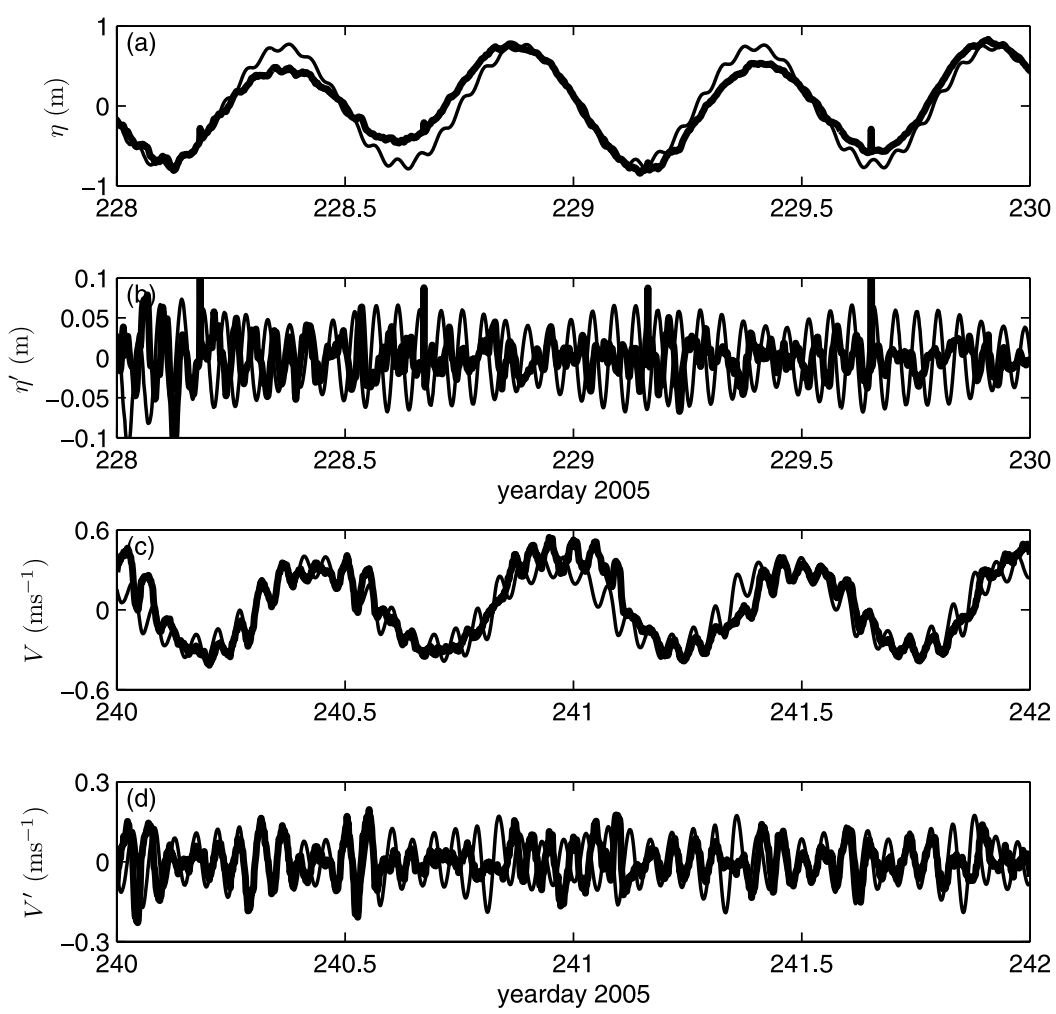

Figure 13. Observed (thick lines) and predicted (thin lines) sea surface elevations at the channel entrance (instrument 4 (Figure 2)) are shown (a) unfiltered and (b) high-pass filtered. North-south velocities in the center of the channel (instrument 8 (Figure 2)) are shown (c) unfiltered and (d) high-pass filtered. Numerical results are for $C_{D}=0.01$ in the channel and coves and $T_{f}=68$ min as in Figure 12 . 

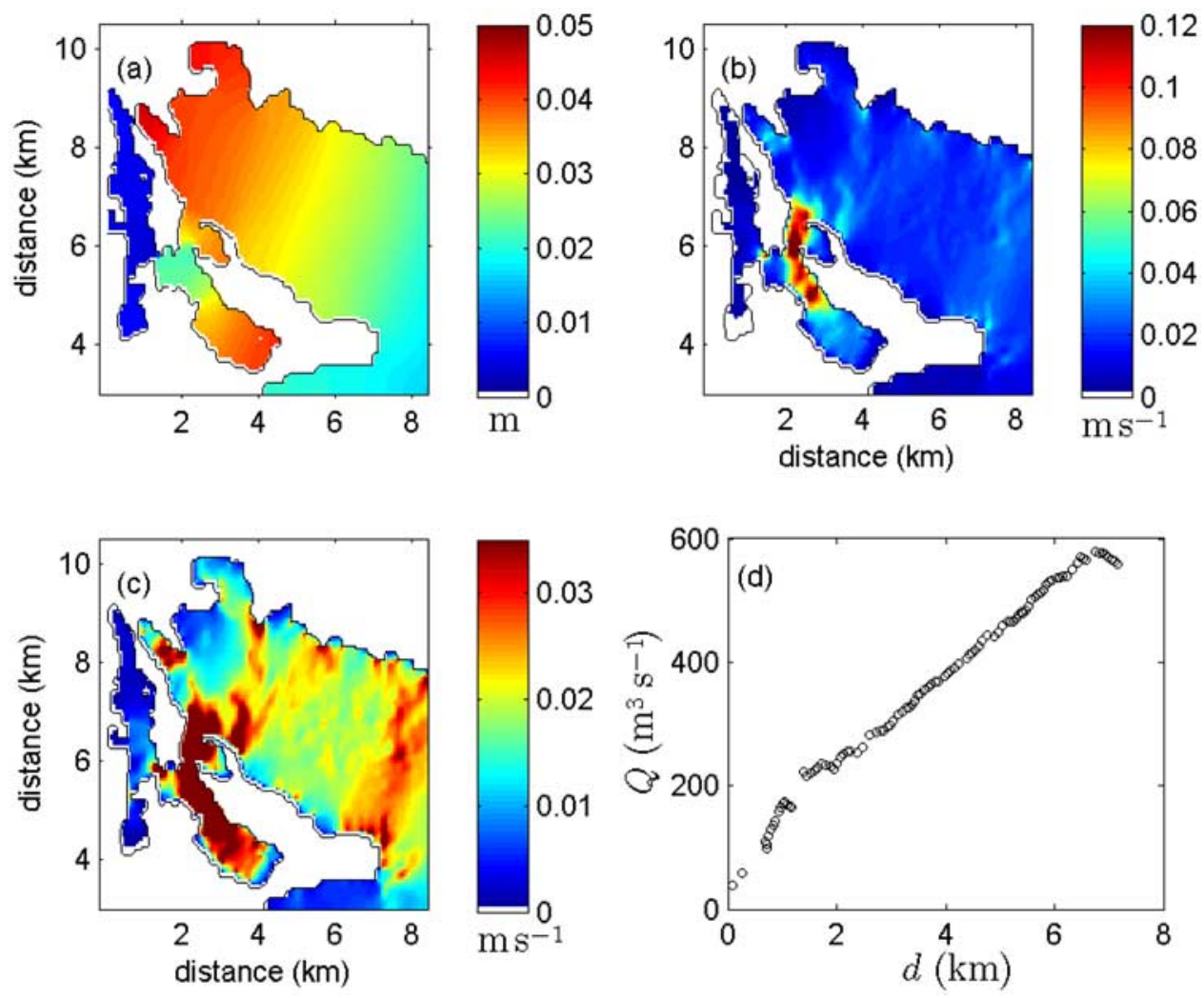

Figure 14. Predicted (a) RMS sea surface elevation, $\sigma_{\eta^{\prime}}$, and (b) velocity, $\sqrt{\sigma_{u^{\prime}}^{2}+\sigma_{v^{\prime}}^{2}}$, distributions in the $0.68-1.08$-cph frequency band for $C_{D}=0.01$ in the channel and coves. (c) The RMS velocities plotted with a different scale to show the along-bay gradient. (d) The RMS transport $Q$ through each lateral cross section based on the RMS velocity $\sqrt{\sigma_{u^{\prime}}^{2}+\sigma_{v^{\prime}}^{2}}$ as a function of distance, $d$, from the head of the bay.

Figures $4 \mathrm{~b}$ and $4 \mathrm{c}$ show the RMS velocity distributions, that is $\sqrt{\sigma_{u^{\prime}}^{2}+\sigma_{v^{\prime}}^{2}}$, with the scale changed in Figure $4 \mathrm{c}$ to show the spatial variation within the bay (so energy levels inside the channel are saturated). The antinode in velocity appears to be located at the seaward side of Sculpin Shoal (Figure 2), in agreement with the position of the node in surface elevation assumed for calculating the quarter-wave resonance period in section 2.4. Energy levels outside of this point fall off. The results also capture the large amplification of the RMS velocities in the channel, which are 1 order of magnitude larger than those inside the bay. The RMS transport over a half cycle of the oscillation from the numerical solutions was calculated along each lateral cross section as

$$
Q=\frac{\sqrt{2}}{\pi} \int h U_{R M S}^{\prime} \mathrm{d} s
$$

where $s$ is the cross-sectional position and the results are shown in Figure 14d as a function of distance from the head of the bay. The results lend support to the existence of a quarter-wave resonance, as there is indeed an increase in RMS transport with distance (up to the assumed node position). Moreover, the RMS transport at the node $(Q \approx$ $600 \mathrm{~m}^{3} \mathrm{~s}^{-1}$ ) is in agreement (within the error bars) of that calculated from the field observations, which were based at a single location.

[30] A similar plot (not shown) for the run using the canonical value for bottom friction coefficient $\left(C_{D}=\right.$ 0.0023 ) demonstrates an increasing amplification of RMS elevation into the channel and coves with maximum oscillation amplitudes at the closed end of Lower South Cove. This trend is not observed in the field data. Indeed, with the canonical friction coefficient, the maximum amplitude of the sea surface oscillation at observation point 3 in Lower South Cove is $0.07 \mathrm{~m}$, about a factor of 2 larger than the amplitudes from the field data and a factor of 1.5 larger than the high-friction run, indicating that the larger coefficient of bottom friction is more appropriate for modeling the flow inside the channel and coves.

\subsection{Phase and Amplitude Response With Friction}

[31] The phase differences (relative to the channel entrance) and dimensionless magnitude of sea surface elevation are shown in Figure 15 for three numerical runs, all forced at the observed oscillation frequency $(f=0.88 \mathrm{cph})$, and using the three different bottom friction coefficients in the channel and coves. For points inside the main bay, distances from the channel entrance were again calculated along the center axis (Figure 5). For the results from the field data and numerical simulations, the phases were 

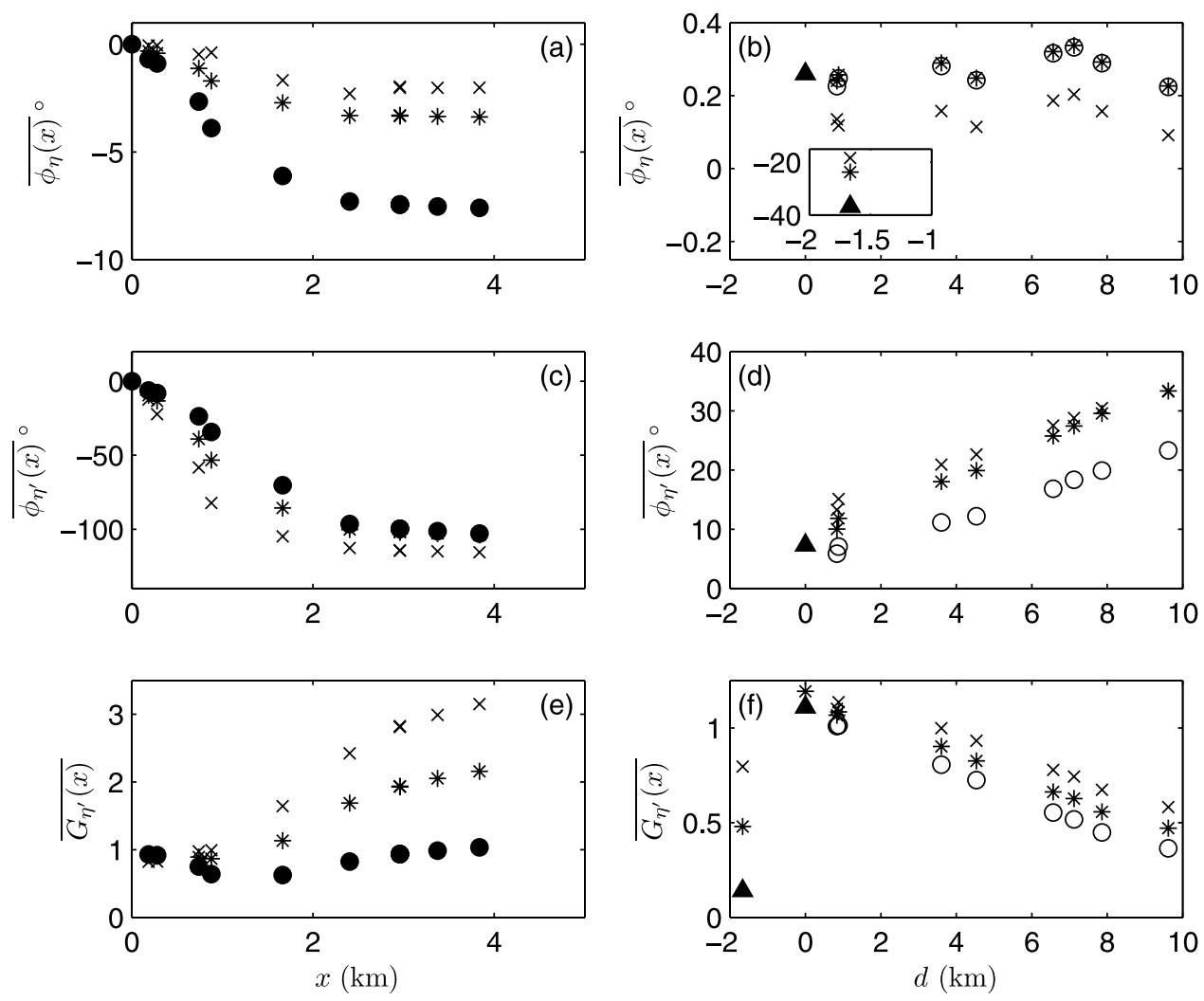

Figure 15. Tidally averaged phases (relative to the entrance to the channel) of sea surface elevation at the (a and b) tidal (M2) frequency and (c and d) oscillation frequency. (e and f) Tidally averaged dimensionless sea surface elevation at the oscillation frequency. Figures $15 \mathrm{a}, 15 \mathrm{c}$, and $15 \mathrm{e}$ show alongchannel position. Figures $15 \mathrm{~b}, 15 \mathrm{~d}$, and $15 \mathrm{f}$ show position in bay. Results from the runs with $C_{D}=0.01$ (closed circles indicate channel and coves, and open circles indicate other), $C_{D}=0.0023$ (asterisks), and $C_{D}=6.1 \times 10^{-5}$ (multiplication signs). The inset in Figure 15b shows the results from Upper South Cove (instrument 1 (Figure 2)).

calculated from the cross spectra. The amplitudes were the RMS elevations in the $0.68-1.08$-cph frequency band made dimensionless by the RMS elevations at the channel entrance, i.e., $\sigma_{\eta^{\prime}}(x) / \sigma_{\eta^{\prime}}(0)$. Figures $5 \mathrm{a}$ and $5 \mathrm{~b}$ show the phase differences at the M2 tidal frequency, while Figures 5d, 5e, and $5 \mathrm{f}$ are the (phase and amplitude) responses at the oscillation frequency. In Figures $5 \mathrm{a}, 5 \mathrm{~b}, 5 \mathrm{c}$, and $5 \mathrm{~d}$, positive values for the phase difference occur only in the main bay and harbor, while phases from inside the channel and coves are negative, consistent with the signal propagating into the bay from outside, along the channel and into Lower South Cove.

[32] The results show a large phase shift along the channel, but points in the interior of the cove (distances of $>2 \mathrm{~km}$ from the entrance) show only a slight phase change, indicating an almost spatially uniform response in the cove interior. Hence, we estimate the effective length of the channel as $L \approx 2000 \mathrm{~m}$. Indeed, the bathymetry (Figure 2) shows there is a widening of the channel into the cove just before the second observation point (the second filled circle from the left in Figure 11). Figures 15a and $15 \mathrm{c}$ show the dependence on bottom friction coefficient for the phases in the channel and coves. Figure 15a for the tidal frequency reveals that increasing the bottom friction increases the phase shift, as expected. However, at the oscillation frequency the opposite trend is observed, i.e., increasing the friction coefficient decreases the phase shift (Figure 15c). This behavior is also captured in the analytical model (see Figure 16 and text below).

[33] The dimensionless amplitudes of the sea surface oscillation are given in Figures 15e and 15f. The open circles, corresponding to points in the main bay, show larger amplitudes close to the head of the bay (and channel entrance), consistent with the quarter-wave oscillation (Figure 15e). The filled circles, corresponding to locations within the channel and Lower South Cove, show an initial decrease in amplitude of the oscillation along the channel. At approximately the halfway point along the channel, the amplitudes start to increase again. This change is also observed in the results from the field data (Figure 4c) and the analytical solution (Figure $10 \mathrm{a}$, solid line) which has a minimum in amplitude at $x / L \approx 0.44$.

[34] The importance of the relative values of the forcing and Helmholtz periods is examined in Figure 16. Figure 16a shows the tidally averaged dimensionless amplitudes at the end of the channel. As before, the amplitudes were calculated as the RMS elevations in a frequency band of width $0.4 \mathrm{cph}$ centered at the forcing frequency, made dimensionless by the RMS elevations (in the same frequency band) at the channel entrance. The symbols show the results from the 

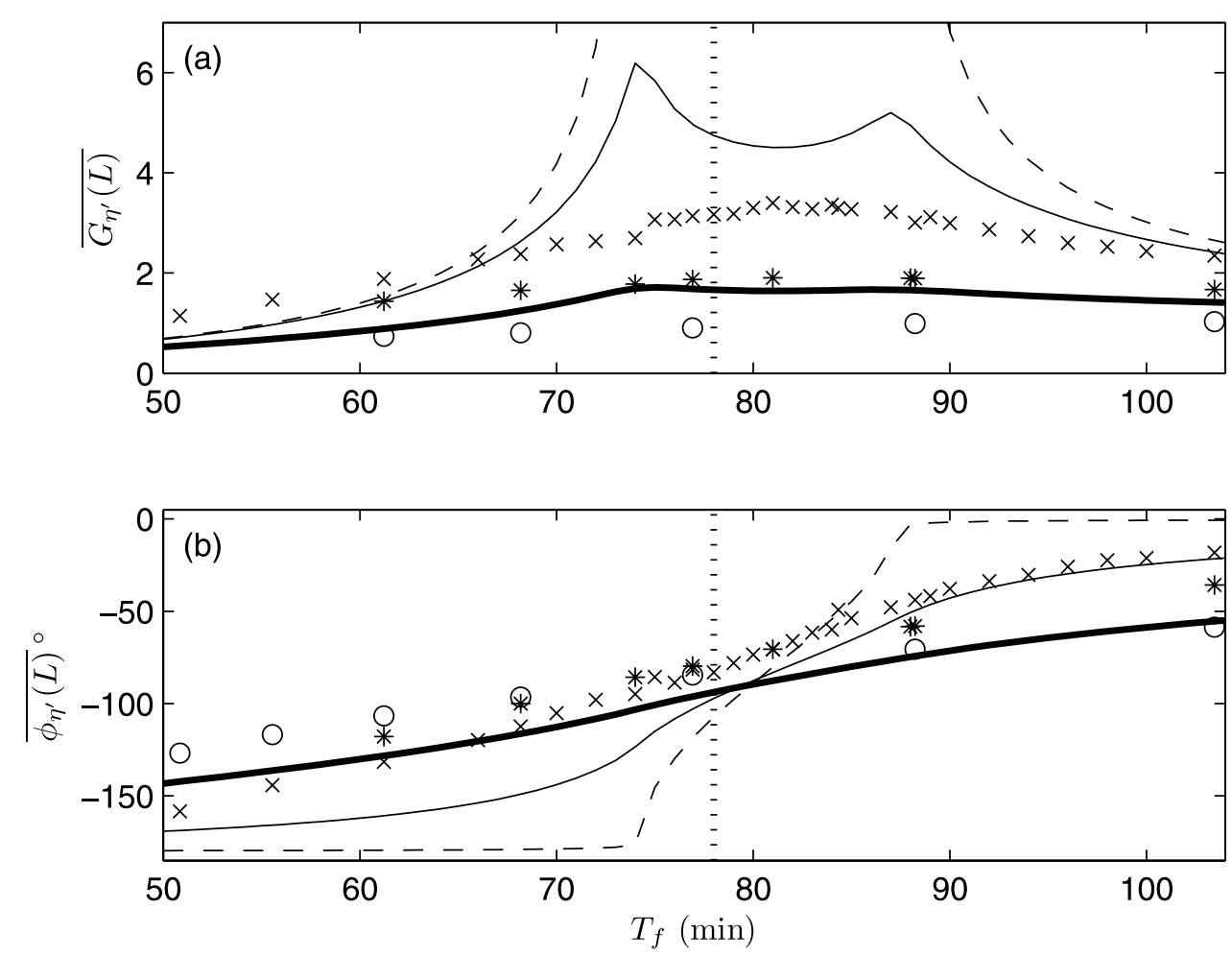

Figure 16. Tidally averaged (a) amplitudes and (b) phases of the sea surface elevation inside the cove $(x=L)$ as a function of the forcing period. The thick, solid, and dashed lines are the analytical solutions with large, canonical, and small bottom friction coefficients inside the channel and coves, respectively. The symbols show the corresponding results from the numerical simulations (open circles show $C_{D}=0.01$, asterisks show $C_{D}=0.0023$, and multiplication signs show $C_{D}=6.1 \times 10^{-5}$ ). The vertical dashed line at $T_{f}=78$ min shows the location at which $T_{f}=T_{H 0}$.

numerical simulations, which exhibit a reduction of amplitude with larger friction coefficients. The numerical system is highly dissipative so the amplitude response curve, even for the runs with low bottom friction, shows only a low broad peak close to the expected resonance period. From the set of runs with low bottom friction coefficient, the maximum amplitude response is observed at $T_{f}=81 \mathrm{~min}$. Hence, we estimate the actual Helmholtz resonance period as $T_{H 0}=$ $78 \mathrm{~min}$ (with variation from 72.4 to $85.1 \mathrm{~min}$ over the tidal cycle) and use this to obtain the analytical solutions.

[35] Figure 16b demonstrates that the phase difference in the cove decreases for larger forcing periods (or lower $\omega_{f}$ ). The frictional dependence changes on either side of the effective Helmholtz period (just greater than $T_{H 0}$ ). For $T_{H 0}>$ $T_{f}$ (or $\omega_{H}<\omega_{f}$ ), increasing the friction coefficient results in lower phase shifts in agreement with the numerical results from Figure 15. This phase response is analogous to that of a single degree of freedom damped harmonic oscillator. In such a system, the two roots of the characteristic equation correspond to the two sides of the system: the "lowfrequency side" (i.e., here $\omega_{H}>\omega_{f}$ ) and the "high-frequency side" (with $\omega_{H}<\omega_{f}$ ). As damping increases on the lowfrequency side, the phase lag increases positively away from zero. Whereas on the high-frequency side, the phase lag moves away (but decreases) from the no-damping value of 180 degrees [e.g., see Thomson, 1971, p. 38]. Conversely, Figure 16 shows for $T_{H 0}<T_{f}$ ( or $\omega_{H}>\omega_{f}$ ), use of the larger friction coefficient results in larger phases shifts. Qualita- tively the numerical simulations exhibit the same phase behavior with friction coefficient as the analytical predictions, although there are quantitative differences between the results.

\subsection{Modulation by the Tide}

[36] The analytical solutions in Figure 16a show a bimodal response in amplitude bracketing the effective Helmholtz frequency. The positions of the two peaks correspond to the Helmholtz periods at high and low tide. When the forcing period is outside the range over which the Helmholtz period varies because of the tide, unsurprisingly the response is much smaller. The bimodality in the analytically predicted response arises as a result of the dependence of Helmholtz period on tidal elevation and is explored further in Figure 17. For $T_{f}<T_{H}$, the largest response is observed at high tide with secondary peaks at low tide (Figure 17e). The largest amplitude response occurs when the forcing period corresponds to the Helmholtz period at high tide (around $74 \mathrm{~min}$ ) and these peaks dominate the tidally averaged value (Figure 17c). Conversely for $T_{f}>T_{H}$, the largest amplitude response shifts to low tide with smaller peaks at high tide (Figure 17i) and the maximum amplitude response occurs when the forcing period equals the Helmholtz period at low tide (Figure 17k). Figure $17 \mathrm{~g}$ demonstrates that when the forcing period is approximately equal to the effective Helmholtz period $(81 \mathrm{~min})$ the peaks at high and low 

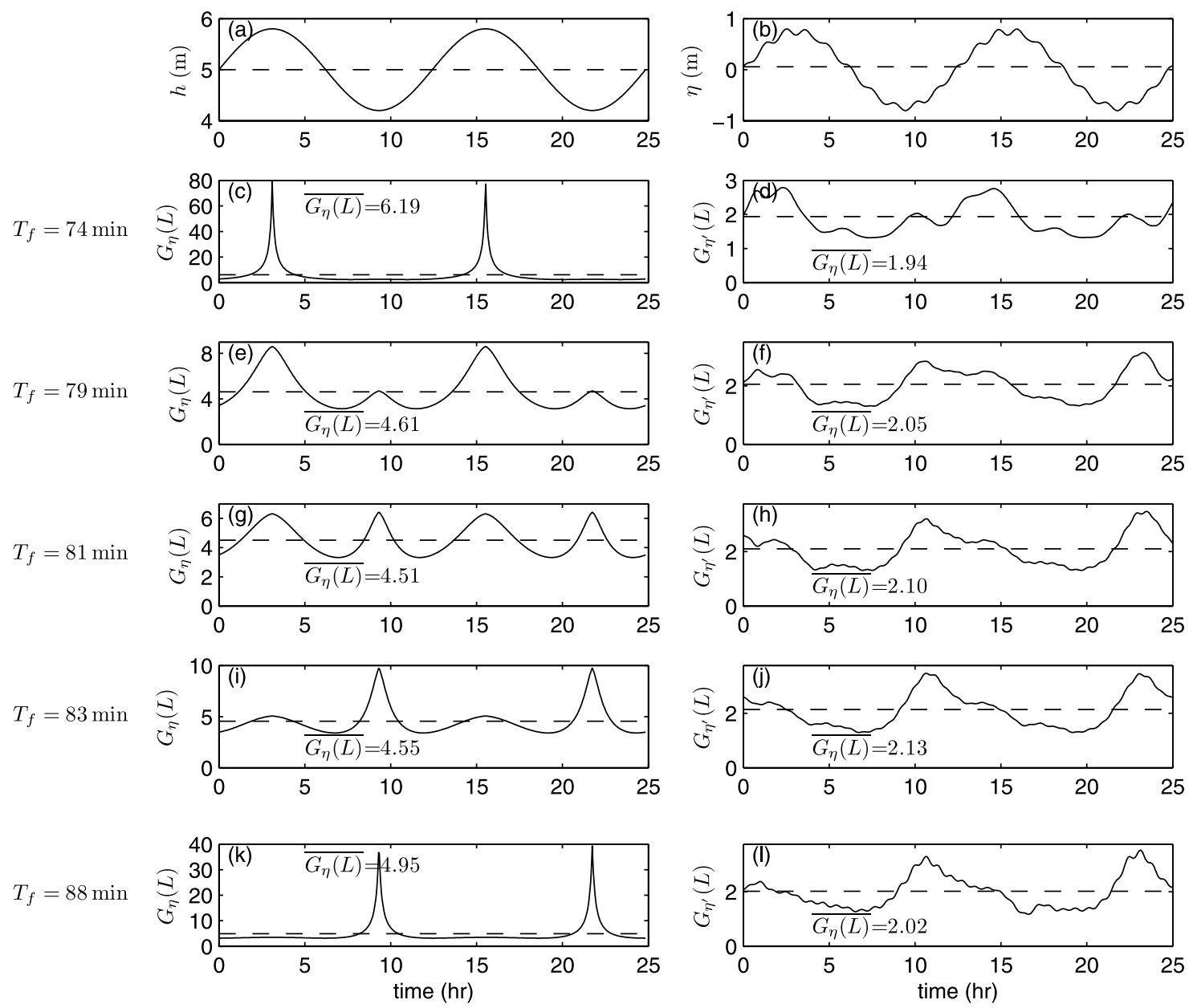

Figure 17. Variation of dimensionless amplitudes at $x=L$ over two M2 tidal cycles. (a, c, e, g, i, and k) Analytical solutions with $C_{D}=0.0023$ and $T_{H}=78 \mathrm{~min}$. (b, d, f, h, j, and l) Corresponding numerical results from the observation point just inside the cove (the fifth closed circle from the right in Figure 11). Tidally averaged values are indicated by the dashed lines. For reference, Figures $17 \mathrm{a}$ and $17 \mathrm{~b}$ show water depths and sea surface elevations from the analytical solutions and numerical run $\left(T_{f}=74 \mathrm{~min}\right)$, respectively.

tide are comparable. The corresponding numerical solutions are depicted in Figures $17 \mathrm{~b}, 17 \mathrm{~d}, 17 \mathrm{f}, 17 \mathrm{~h}, 17 \mathrm{j}$, and 171. These dimensionless amplitudes over the tidal cycle were calculated from the spectra of overlapping data segments of length 2.5 hours. The results also show a shift in location of the largest peaks from high to low tide between Figures $17 \mathrm{~b}$ and $17 \mathrm{f}$. However, the peaks in $G_{\eta^{\prime}}(L)$ are both much smaller and much broader than in the analytical solutions. We note that this broadness is due, in part, to smearing arising from the use of overlapping 2.5-hour segments. In addition, at the high and low points in the tidal cycle, the friction in the analytical solutions is very small which contributes to the (unrealistically) large amplitude responses. Friction is larger in the numerical solutions at low and high tide, partly owing to the harmonics of the M2 tidal forcing, which are generated in the numerical model but are not included in the analytical model.

[37] Figures 18a, 18c, 18d, 18f, and 18h show the modulation of relevant quantities over the tidal cycle for $C_{D}=0.01$ based on the analytical solutions. The water depth outside the channel follows the sinusoidal dependence illustrated in Figure 18a. The variation of the Helmholtz period about the base period is plotted in Figure $18 \mathrm{c}$. Observe that the high (low) tide corresponds to smaller (larger) Helmholtz periods. The friction term is given in Figure 18d. The amplitude (Figure 18f) and phase (Figure 18h) plots are consistent with the behavior predicted by (28) and (29) and discussed in section 3. There is a 12.4-hour modulation owing to the variation of Helmholtz period over the tidal cycle and a 6-hour modulation because of the variation of friction. $T_{H}>T_{f}$ (or $\omega_{H}<\omega_{f}$ ) throughout the tidal cycle and therefore the phase falls between $-90^{\circ}$ and $-180^{\circ}$. A second example using $T_{H 0}=$ $70 \mathrm{~min}$ is also included to illustrate this phase modulation more clearly and is shown by the thick dashed line (with tidally averaged values shown by the dotted line). In this latter case, for the 6-hour period surrounding high tide, $T_{H}<T_{f}$ (or $\omega_{H}>\omega_{f}$ ). Thus, the phase falls between $0^{\circ}$ and $-90^{\circ}$ as predicted by 28 . Conversely for the 6 -hour period surrounding low tide, $T_{H}>T_{f}$ (or $\omega_{H}<\omega_{f}$ ) and so the phase falls between $-90^{\circ}$ and $-180^{\circ}$. The key role played by 

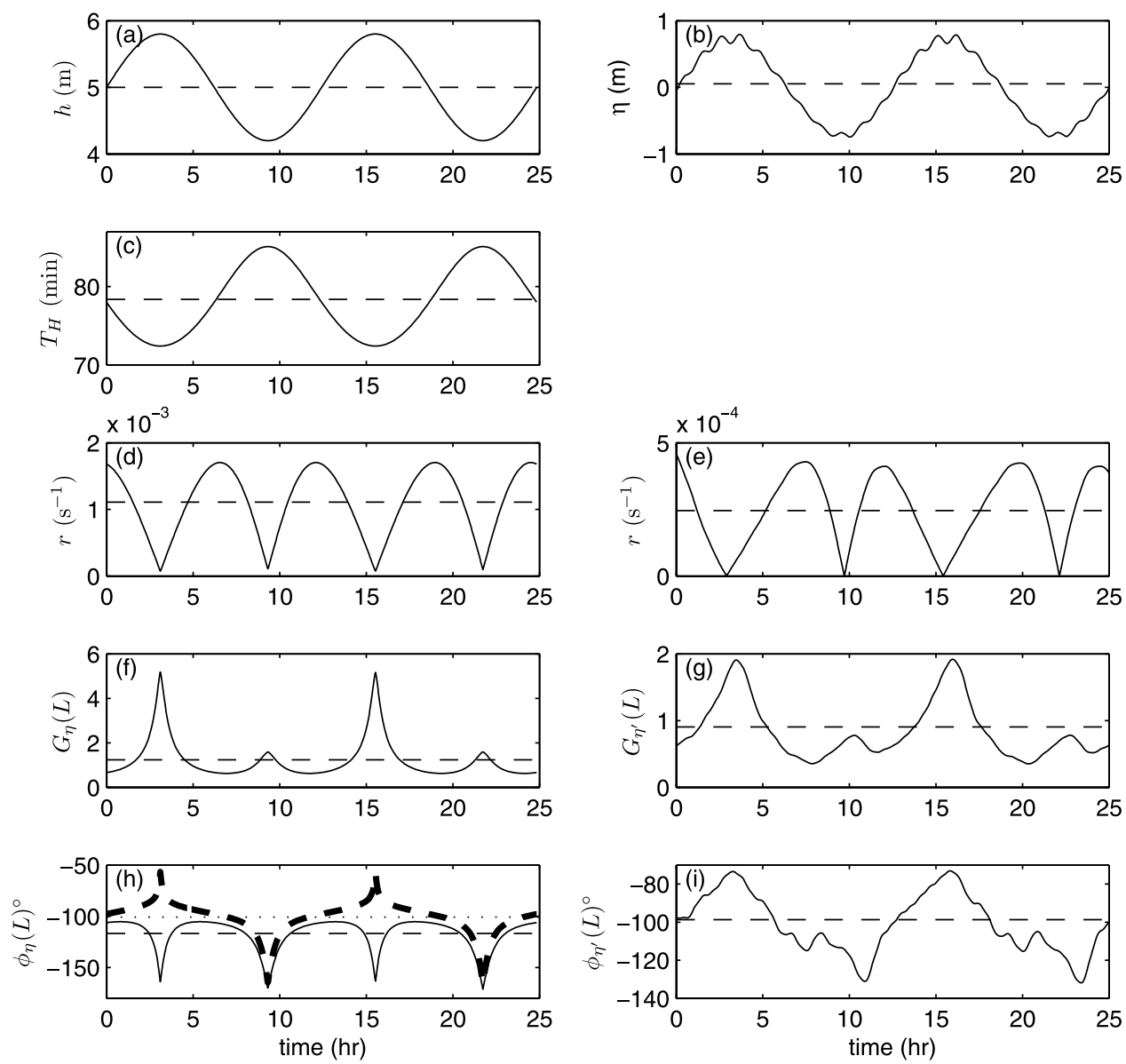

Figure 18. Variation of (a) water depth, (b) sea surface elevation, (c) Helmholtz period (unknown in the numerical simulations), ( $\mathrm{d}$ and e) friction, (f and $\mathrm{g}$ ) amplitudes at $x=L$, and (h and i) phases at $x=$ L. $C_{D}=0.01$, and $T_{f}=68 \mathrm{~min}$. Results from the analytical solution over two $\mathrm{M} 2$ tidal cycles are shown (Figures 18a, 18c, 18d, 18f, and 18h). The corresponding numerical results from the observation point just inside the cove are shown as well (Figures $18 \mathrm{~b}, 18 \mathrm{e}, 18 \mathrm{~g}$, and $18 \mathrm{i}$ ). Tidally averaged values are shown by the dashed line. An additional example of phase modulation with $T_{H 0}=70 \mathrm{~min}$ is shown by the dot-dash line in Figure $18 \mathrm{~h}$ with the dotted line indicating the tidally averaged value.

friction is again apparent with large oscillation amplitudes occurring in response to the friction term decreasing to its minimum value at slack tide when the tidal velocities in the model are lowest. Numerical solutions (Figures 18b, 18e, $18 \mathrm{f}, 18 \mathrm{~g}, 18 \mathrm{~h}$, and 18i) from inside the cove again show qualitative agreement, but as before the magnitudes are different. The maximum $r$-value in the numerical solutions (Figure 18e) is approximately a factor of 4 smaller than in the analytical model (Figure 18d). This difference is because the tidal velocities in the depth-averaged numerical model are smaller than those used in the analytical solution. Furthermore, the depth $h$ at the observation point used from the numerical solutions is slightly less than that used in the analytical solution, which uses constant $h=5 \mathrm{~m}$. The phase differences in the cove (relative to the channel entrance) and dimensionless amplitudes over the tidal cycle were calculated from the spectra of overlapping data segments of length 2.5 hours. The 12.4-hour and 6-hour variability cannot be discerned in the noisier field data (Figure 7), probably because it is obscured by contributions from the other tidal constituents.

\subsection{Comparison With Field Observations}

[38] Finally, the numerical results and analytical predictions for phase and amplitude are compared with the field observations in Figure 19. At the tidal frequency (Figures 19a and 19b), the agreement for phase is excellent everywhere, even though only a single tidal constituent was used to force the numerical model. At the oscillation frequency (Figures 19c and 19d), the observations and numerical results agree well for locations inside the bay and channel. The analytical predictions follow the same qualitative pattern, but the gradient of phase shift along the channel is slightly steeper, which is a consequence of the theory overpredicting the phase differences (by up to $40^{\circ}$ in some cases). The amplitudes from the analytical results match well in the channel but the amplitudes in the cove are larger than those observed (Figure 19e). Possible explanations for this are discussed below. We note that the observed phases in the cove can be obtained in the theoretical model by using a Helmholtz period of $T_{H 0}=70 \mathrm{~min}$ (dashed line), although this leads to unrealistic oscillation amplitudes. 

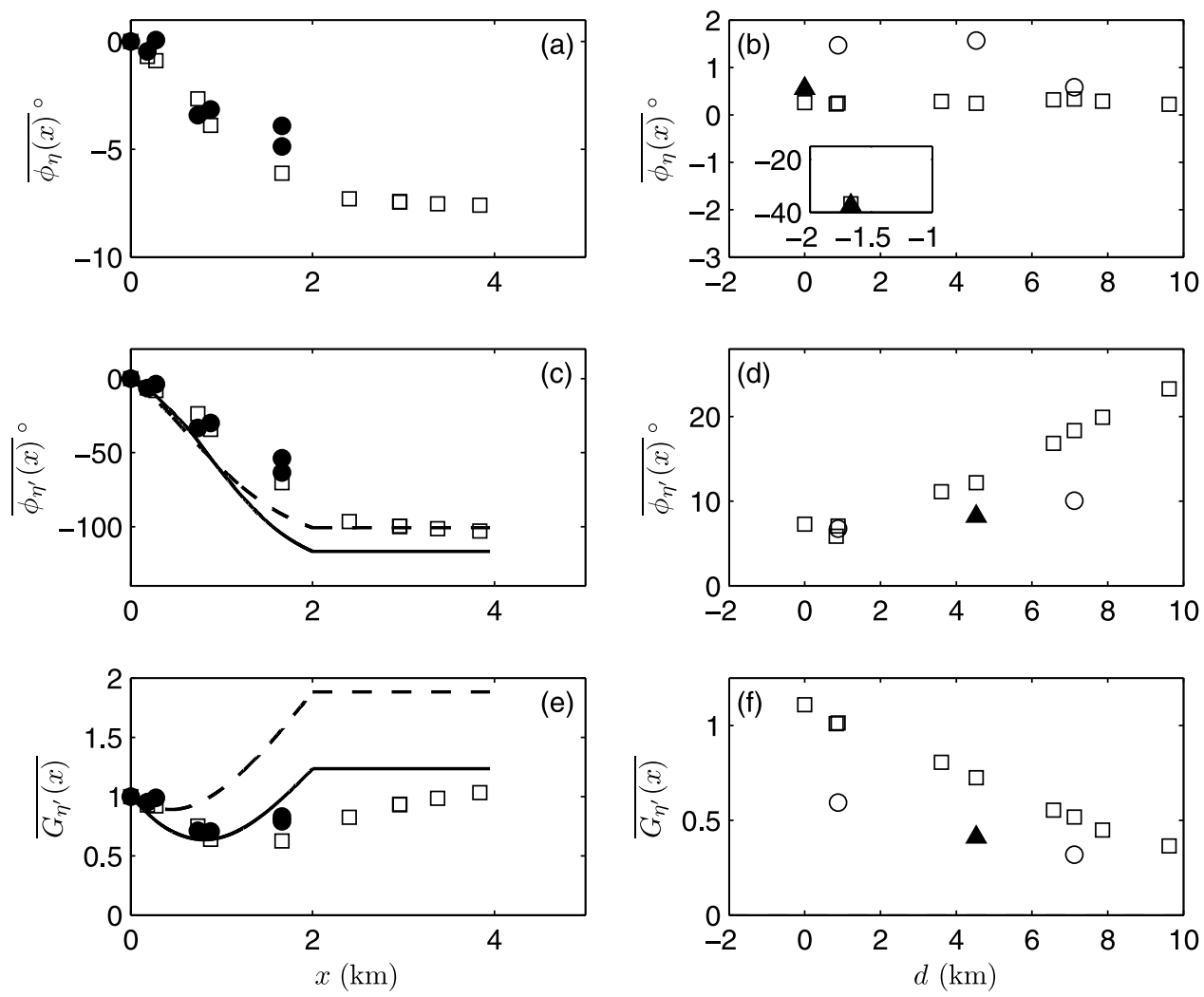

Figure 19. ( $a$ and b) Tidally averaged phase differences (relative to the channel entrance) at the tidal (M2) frequency band. (c and d) Phase differences and (e and f) dimensionless amplitudes at the oscillation frequency. Positions in the channel and coves are shown (Figures 19a, 19c, and 19e). Positions in the bay are also shown (Figures 19b, 19d, and 19f). Numerical results (squares), field data (closed circles, open circles, and closed triangles), and analytical solutions using $T_{H 0}=78$ min (solid line) and $T_{H 0}=70 \mathrm{~min}$ (dashed line). In all solutions, $T_{f}=68 \mathrm{~min}$ and $C_{D}=0.01$. The inset in Figure $19 \mathrm{~b}$ shows the results from Upper South Cove (instrument 1 (Figure 2)).

\subsection{Discussion}

[39] The analytical model uses a greatly simplified geometry, assuming uniform depth everywhere in the channel and cove, and is one-dimensional and linear. However, despite its highly idealized nature the model appears to qualitatively capture the flow behavior seen in the numerical model. In particular, the model correctly predicts the dependence of the phase shift along the channel on bottom friction coefficient. It captures the modulation of phase and amplitude throughout the tidal cycle and the variation of these quantities along the channel. There are however, some quantitative discrepancies between the analytical model results and both the numerical results and field observations. The phase differences (relative to the channel entrance) are overpredicted (by up to $40^{\circ}$ in some locations). The amplitudes of oscillations match reasonably well with observed tidally averaged values $75 \%$ of those predicted (Figure 19).

[40] The lack of lateral friction in the analytical model appears not to contribute significantly to the discrepancies in phase. Indeed, changing the horizontal eddy viscosity in the channel and coves by a factor of 10 in the numerical simulations results in less than $1^{\circ}$ changes in phase within the cove. We suggest that the lack of nonlinear effects most likely contributes to some part of the phase discrepancies. The simplified geometry in the analytical model is also expected to play a role. The numerical results lend support to these hypotheses as the simulations represent the geometry accurately and include nonlinear effects and the results match the observations well.

\section{Conclusions}

[41] Observations have been presented showing a double resonance. The first, an oscillation in sea surface elevation and flow velocity with a period of around 1 hour, has an energy distribution, which was shown to be consistent with a quarter-wave resonance of the bay. Moreover, the occurrence of large amplitude events of sea level variance in the 1-hour band in Lunenburg Bay are shown to be often coherent with similar sea level variance events at other locations. The peak of the quarter-wave resonance is broadbanded and sufficiently close in frequency to the Helmholtz frequency of a connected cove to excite a Helmholtz resonance in the cove. The interaction between the two resonances leads to a strong modulation of the tidal currents in the connecting channel. An analytical model of the interaction shows that the $\sim 1$-hour motion in the channel is a standing wave and gives good qualitative and reasonable quantitative agreement with results from a numerical model and the field data. The quantitative differences are 
attributed in part to lack of nonlinear effects and the simplified geometry in the analytical model. As far as we are aware, this is the first model exploring the phase response of a Helmholtz oscillation to varying friction coefficients. While the results presented here are specific to the geometry of Lunenburg Bay, tidal channels connecting bays and coves are common features of the coastal environment, and one expects that similar flow dynamics exist elsewhere.

[42] Acknowledgments. We thank Ryan Mulligan for data from the shoal experiment, Doug Schillinger and Walter Judge for technical assistance, and the rest of the field crew of the Ustar. Many thanks to Keith Thompson for useful discussions. This work was funded by grants from the Canadian Foundation for Climate and Atmospheric Sciences, the Canada Foundation for Innovation, and the Natural Sciences and Engineering Research Council of Canada. Use of Delft3D was provided through the Office of Naval Research Delft3D Community Model Development Project. Comments from J. A. Battjes and an anonymous reviewer helped to improve the paper.

\section{References}

Defant, A. (1961), Physical Oceanography, vol. 2, Elsevier, New York. de Jong, M. P. C., and J. A. Battjes (2004), Low-frequency sea waves generated by atmospheric convection cells, J. Geophys. Res., 109, C01011, doi:10.1029/2003JC001931.

de Jong, M. P. C., L. H. Holthuijsen, and J. A. Battjes (2003), Generation of seiches by cold fronts over the southern North Sea, J. Geophys. Res., 108(C4), 3117, doi:10.1029/2002JC001422.

Dronkers, J. J. (1964), Tidal Computations in Rivers and Coastal Waters, Elsevier, Amsterdam.

Eichler, T., and W. Higgins (2006), Climatology and ENSO-related variability of North American extratropical cyclone activity, J. Clim., 19(10), $2076-2093$.

Elias, E., D. Walstra, J. Roelvink, M. Stive, and M. Klein (2000), Hydrodynamic validation of Delft3D with field measurements at Egmond, in Coastal Engineering, pp. 2714-2727, Am. Soc. of Civ. Eng., Sydney, Australia.
Gomis, D., S. Monserrat, and J. Tintoré (1993), Pressure-forced seiches of large amplitude in inlets of the Balearic Islands, J. Geophys. Res., 98, $14,437-14,445$.

Green, T. (1992), Liquid oscillations in a basin with varying surface-area, Phys. Fluids A, 4(3), 630-632.

Lee, J. (1971), Wave-induced oscillations in harbours of arbitrary geometry, J. Fluid Mech., 45, 375-394.

Lesser, G. R., J. A. Roelvink, J. van Kester, and G. S. Stelling (2004), Development and validation of a three-dimensional morphological model, Coastal Eng., 51(8-9), 883-915.

Maas, L. R. M. (1997), On the nonlinear Helmholtz response of almostenclosed tidal basins with sloping bottoms, J. Fluid Mech., 349, 361380.

Miles, J. W. (1974), Harbor seiching, Annu. Rev. Fluid Mech., 6, 17-35.

Miles, J. W., and Y. K. Lee (1975), Helmholtz resonance of harbors, J. Fluid Mech., 67, 445-464.

Mulligan, R. P., A. J. Bowen, and A. E. Hay (2006), Waves and flows over a bedrock shoal, Eos Trans. AGU, 87, Ocean Sci. Meet. Suppl., Abstract OS11B-03.

Munk, W., F. Snodgrass, and C. Carrier (1956), Edge waves on the continental shelf, Science, 123(3187), 127-132.

Platzman, G. W. (1972), Two-dimensional free oscillations in natural basins, J. Phys. Oceanogr., 2, 117-138.

Priestley, M. B. (1981), Spectral Analysis and Time Series, vol. 1-2, Elsevier, London.

Sheng, J. Y., and L. Wang (2004), Numerical study of tidal circulation and nonlinear dynamics in Lunenburg Bay, Nova Scotia, J. Geophys. Res., 109, C10018, doi:10.1029/2004JC002404.

Sturley, D. R. M., and A. J. Bowen (1996), A model for contaminant transport in Lunenburg Bay, Nova Scotia, Sci. Total Environ., $179(1-3), 161-172$

Thomson, W. T. (1971), Vibration Theory and Applications, Allen and Unwin, London.

Tintoré, J., D. Gomis, S. Alonso, and D. P. Wang (1988), A theoreticalstudy of large sea-level oscillations in the western Mediterranean, J. Geophys. Res., 93, 10,797-10,803.

A. J. Bowen, A. E. Hay, and J. C. Mullarney, Department of Oceanography, Dalhousie University, 1355 Oxford Street, Halifax, NS, Canada B3H 4J1. (julia.mullarney@phys.ocean.dal.ca) 